\title{
Daily hypoxia forecasting and uncertainty assessment via Bayesian mechanistic model for the Northern Gulf of Mexico
}

Alexey Katin ${ }^{1}$, Dario Del Giudice ${ }^{1}$, Daniel R. Obenour ${ }^{1}$

${ }^{1}$ Department of Civil, Construction, \& Environmental Engineering, North Carolina State University, Raleigh, 27606, USA

Supporting information 


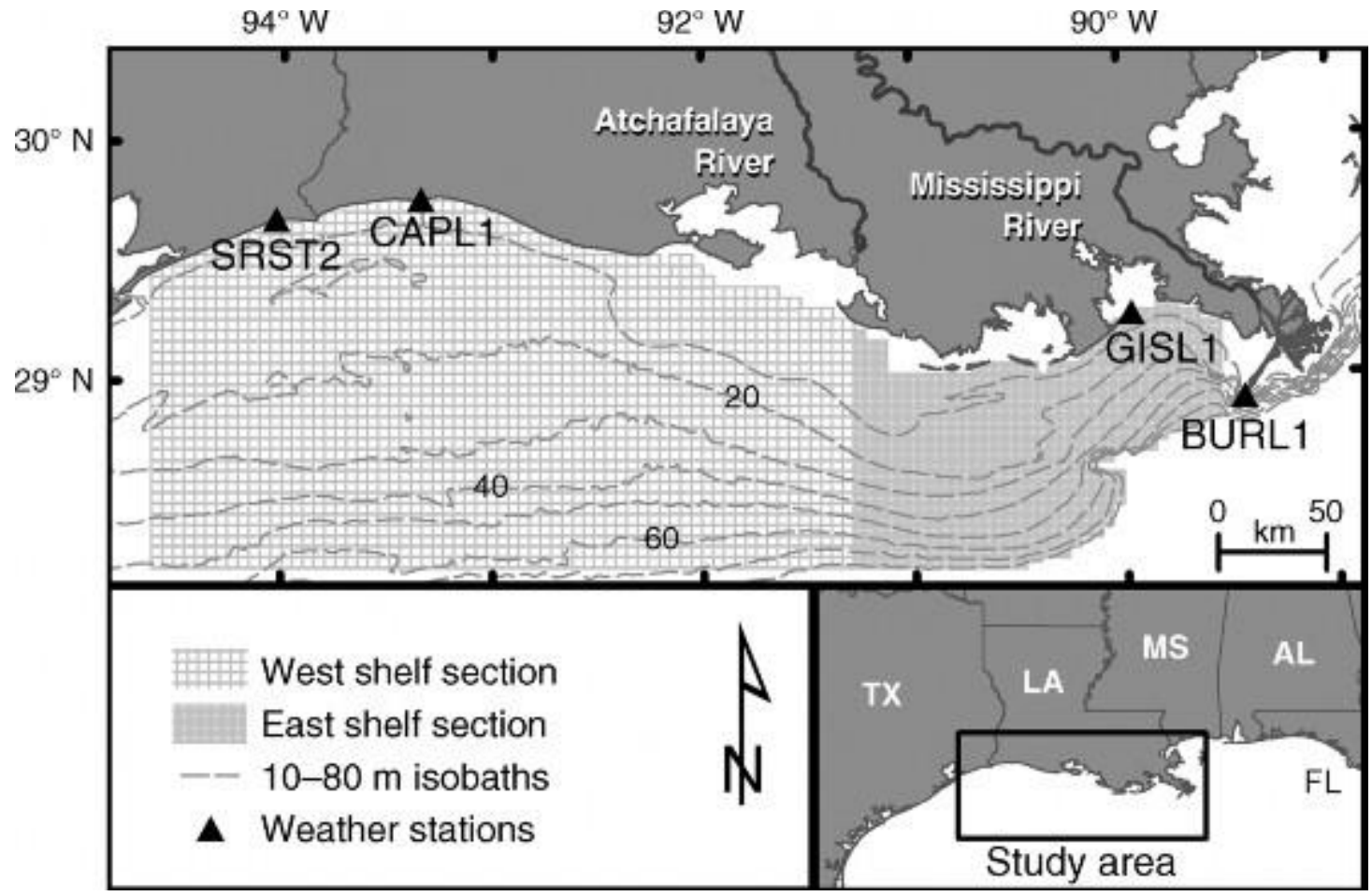

Figure S1: Map of the study area located in the Northern Gulf of Mexico (Obenour et al., 2015). 


\section{S2 Bias adjustment for model predictions in June}

Preliminary analysis indicated that hindcasted BWDO was somewhat lower than observations for the west section of the shelf in June. This bias remained after conversion of BWDO to HA. A linear regression with day number (June 1 - June 30) as the predictor was used to estimate a bias adjustment, defined as difference between average observed and hindcasted BWDO divided by hindcasted BWDO. The resulting regression indicates a gradual decline in bias from the beginning of June $(+20 \%)$ to the end of June (+0\%) (Fig. S2.1). The bias adjustment increases the $R^{2}$ of relationship between observed and hindcasted BWDO from -0.16 to 0.36 (Fig. S2.2), and between observed and hindcasted HA from -0.16 to 0.45 (Fig. S2.3).

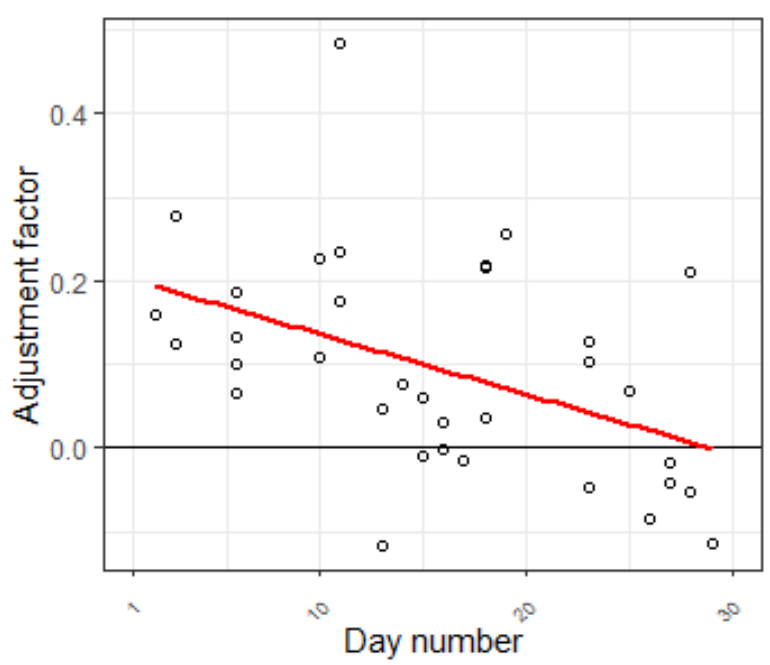

Figure S2.1: Bias adjustment factor vs day number for the west section in June with red line showing the regression fit with intercept term (0.208) and slope (-0.007). The adjusted $R^{2}$ of this regression is 0.18 . 


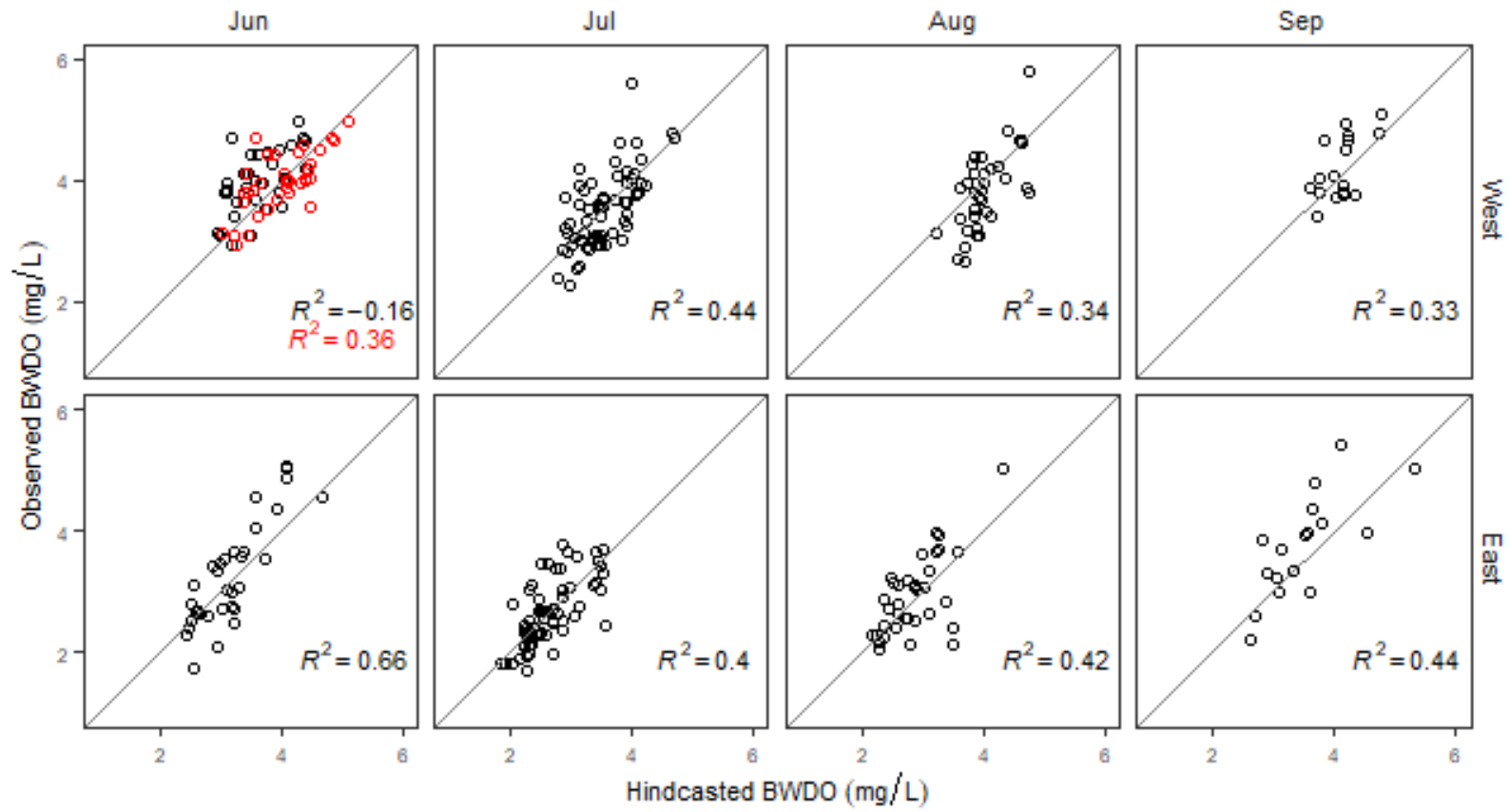

Figure S2.2: Month by month comparison of observed with hindcasted (black) and bias-adjusted hindcasted (red) averaged BWDO in the west and east sections. Diagonal lines represent perfect prediction.

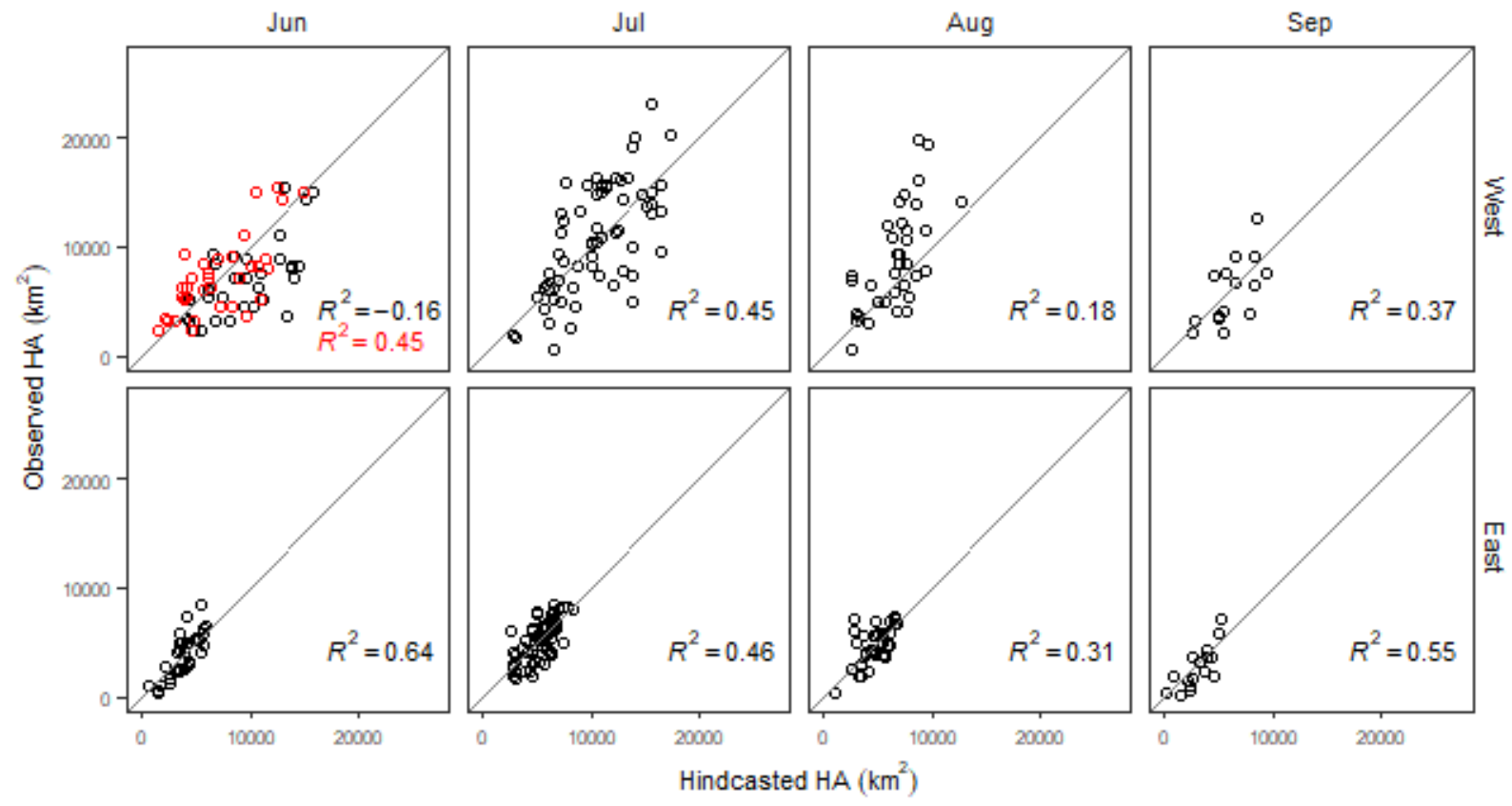

Figure S2.3: Month by month comparison of observed with hindcasted (black) and bias-adjusted hindcasted (red) averaged HA in the west and east sections. Diagonal lines represent perfect prediction. 
S3 Linear regressions for predicting June-September MAR flow and loading

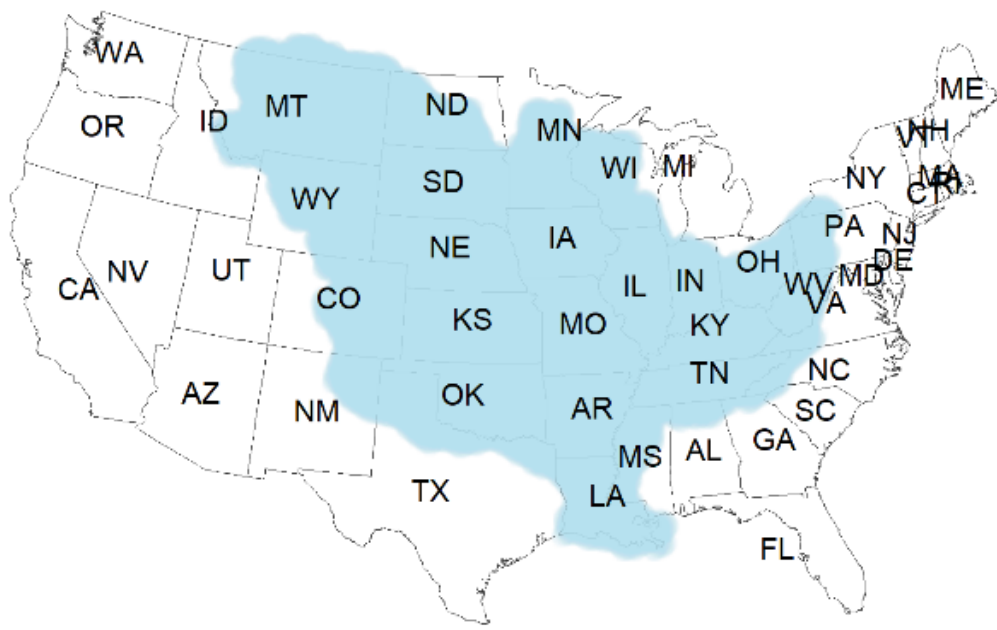

Figure S3.1: Map of MAR Basin (light blue filled) used in estimation monthly precipitation and temperature. 

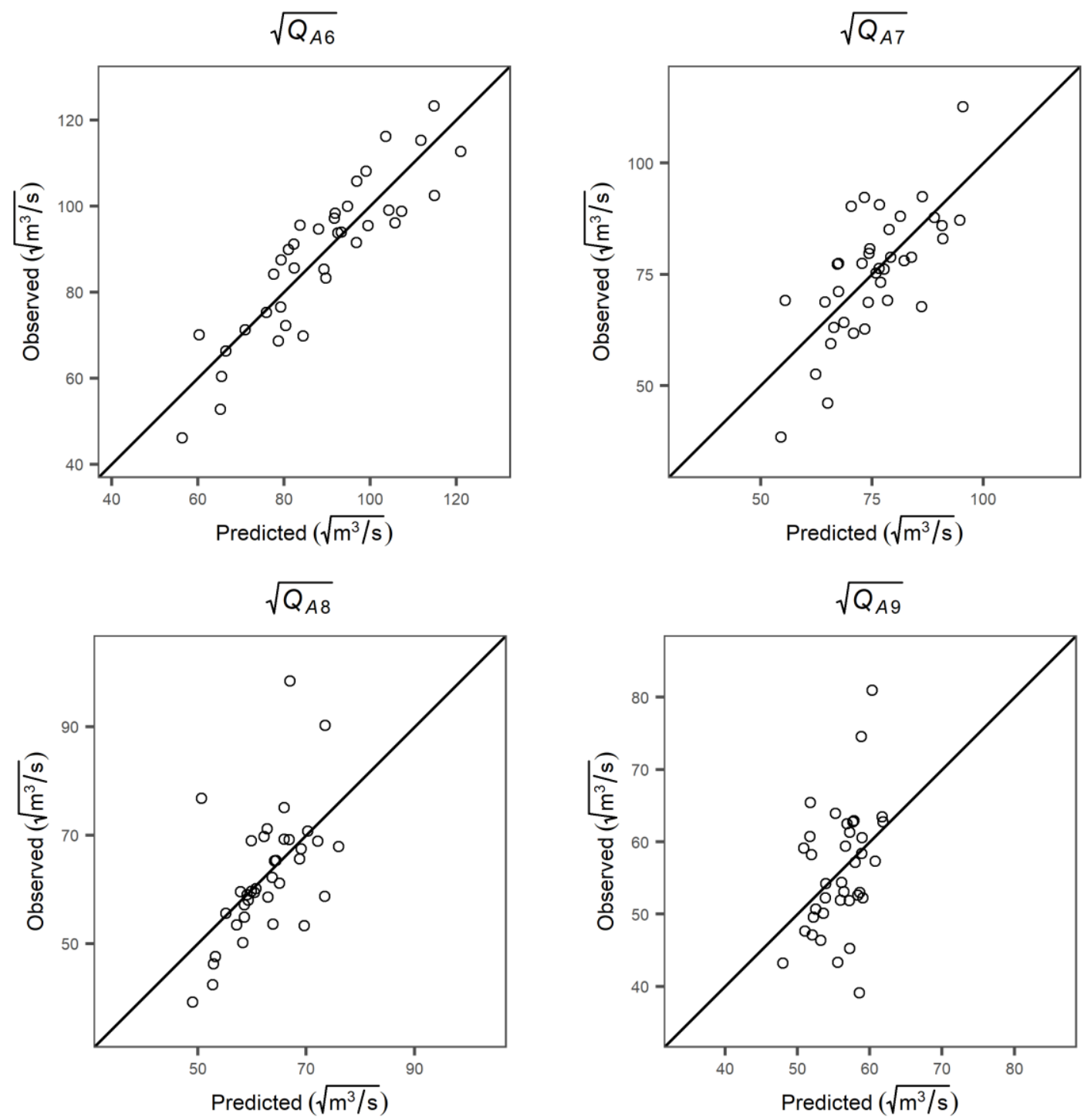

Figure S3.2: Observed versus predicted by regressions square-root transformed Atchafalaya River monthly average discharge. Subscript numbers indicate months. 

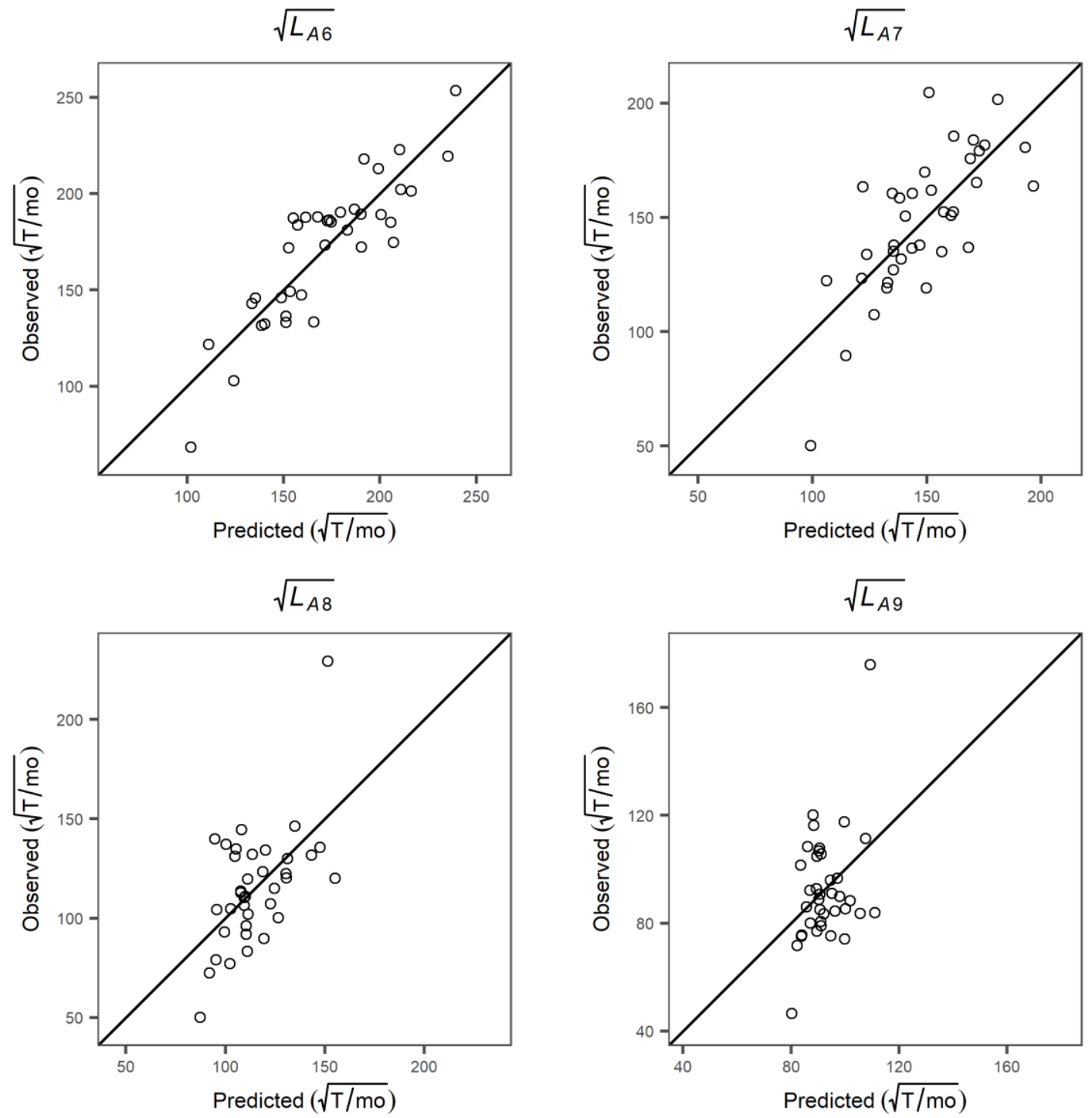

Figure S3.3: Observed versus predicted by regressions square-root transformed Atchafalaya River monthly average nitrogen loading. Subscript numbers indicate months. 

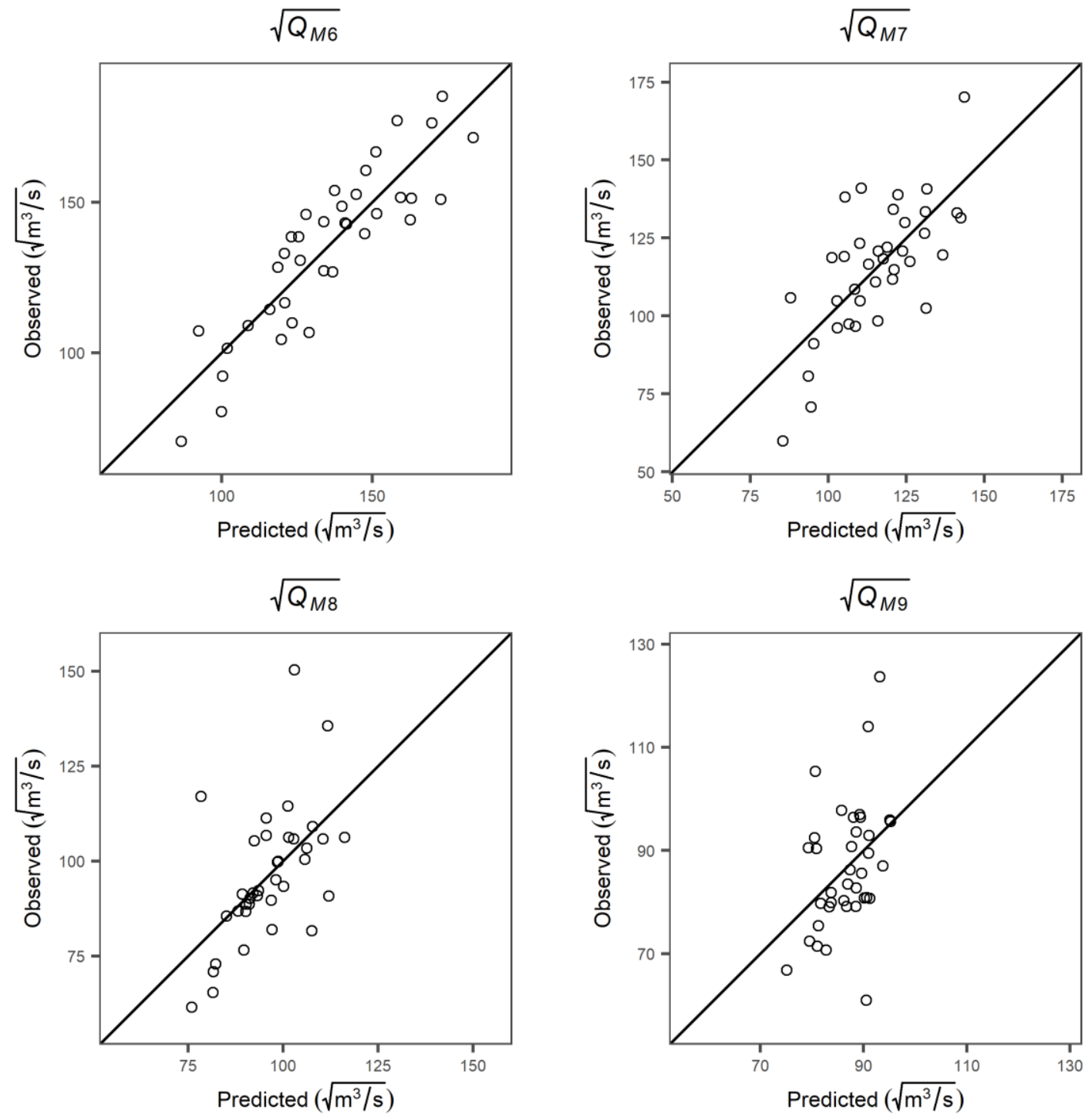

Figure S3.4: Observed versus predicted by regressions square-root transformed Mississippi River monthly average discharge. Subscript numbers indicate months. 

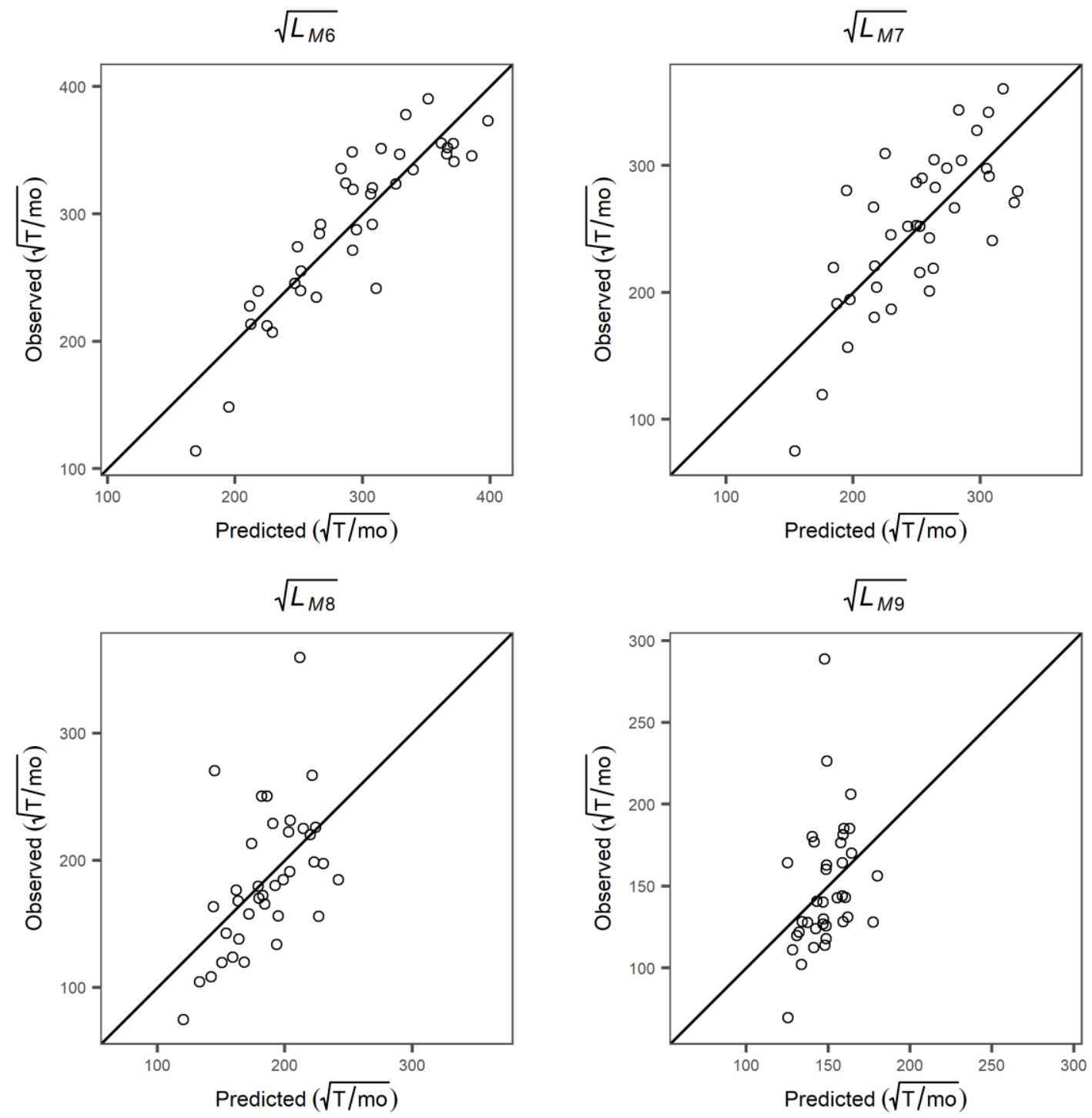

Figure S3.5: Observed versus predicted by regressions square-root transformed Mississippi River monthly average nitrogen loading. Subscript numbers indicate months. 
Table S3.1: Predicted by the regressions (pre) and observed (obs) monthly Atchafalaya River discharge and nitrogen loading

\begin{tabular}{|c|c|c|c|c|c|c|c|c|c|c|c|c|c|c|c|c|}
\hline \multirow{4}{*}{ Year } & \multicolumn{16}{|c|}{ Atchafalaya River } \\
\hline & \multicolumn{8}{|c|}{ Discharge $\left(\mathrm{m}^{3} / \mathrm{s}\right)$} & \multicolumn{8}{|c|}{ Loading (t/mo) } \\
\hline & \multicolumn{2}{|c|}{ Jun } & \multicolumn{2}{|c|}{ Jul } & \multicolumn{2}{|c|}{ Aug } & \multicolumn{2}{|c|}{ Sep } & \multicolumn{2}{|c|}{ Jun } & \multicolumn{2}{|c|}{ Jul } & \multicolumn{2}{|c|}{ Aug } & \multicolumn{2}{|c|}{ Sep } \\
\hline & pre & obs & pre & obs & pre & obs & pre & obs & pre & obs & pre & obs & pre & obs & pre & obs \\
\hline 1980 & 6267 & 5860 & 4719 & 4130 & 3429 & 3270 & 3466 & 3410 & 23542 & 22304 & 18328 & 16168 & 11974 & 12183 & 8138 & 11426 \\
\hline 1981 & 7743 & 8970 & 5517 & 6360 & 3872 & 4870 & 2699 & 3390 & 28095 & 35351 & 20638 & 25812 & 10072 & 18866 & 7413 & 11767 \\
\hline 1982 & 8692 & 8820 & 7035 & 6220 & 4148 & 4270 & 3805 & 4030 & 32188 & 36213 & 23060 & 26246 & 10964 & 17236 & 7757 & 14451 \\
\hline 1983 & 13167 & 15200 & 6754 & 6100 & 5385 & 3450 & 3088 & 1880 & 57267 & 64260 & 38645 & 26815 & 24069 & 14452 & 12311 & 7060 \\
\hline 1984 & 8963 & 9990 & 5293 & 6010 & 4229 & 3740 & 3273 & 2050 & 39694 & 45366 & 28585 & 30918 & 20541 & 17382 & 11149 & 7000 \\
\hline 1985 & 5756 & 5670 & 4409 & 3980 & 3270 & 2860 & 3360 & 3270 & 22140 & 21315 & 17544 & 14186 & 12186 & 8462 & 8218 & 8258 \\
\hline 1986 & 6284 & 7650 & 4550 & 5070 & 3434 & 3010 & 2756 & 2570 & 26063 & 35274 & 19776 & 22708 & 11990 & 11333 & 8144 & 7891 \\
\hline 1987 & 6180 & 4710 & 5014 & 3820 & 3401 & 2520 & 2725 & 2460 & 22852 & 18584 & 17639 & 14776 & 9903 & 8680 & 7347 & 7414 \\
\hline 1988 & 3175 & 2130 & 2972 & 1480 & 2406 & 1540 & 3427 & 1530 & 10378 & 4674 & 9845 & 2525 & 7629 & 2524 & 6431 & 2164 \\
\hline 1989 & 6758 & 8310 & 5372 & 8520 & 3579 & 3560 & 3340 & 3960 & 19207 & 17314 & 15313 & 17902 & 8453 & 5270 & 6770 & 5147 \\
\hline 1990 & 10735 & 13500 & 8260 & 6890 & 4724 & 4310 & 2673 & 3690 & 34861 & 36813 & 24760 & 23264 & 12379 & 14335 & 8289 & 11185 \\
\hline 1991 & 11184 & 9240 & 7418 & 4590 & 4849 & 2840 & 2874 & 2510 & 40243 & 35772 & 28265 & 18731 & 16010 & 10062 & 9599 & 8104 \\
\hline 1992 & 3628 & 4920 & 3084 & 4780 & 2568 & 5900 & 2587 & 3500 & 12358 & 14835 & 11260 & 14967 & 8974 & 19610 & 6980 & 10322 \\
\hline 1993 & 9887 & 9120 & 5878 & 8220 & 4490 & 9690 & 3639 & 6560 & 46759 & 40591 & 32790 & 40674 & 22914 & 52641 & 11934 & 30940 \\
\hline 1994 & 6458 & 5220 & 4147 & 4740 & 3489 & 3480 & 3436 & 2810 & 22887 & 17737 & 18342 & 18275 & 15049 & 11522 & 9259 & 7145 \\
\hline 1995 & 12495 & 13300 & 8971 & 7610 & 5202 & 4750 & 3146 & 2960 & 44192 & 49676 & 29920 & 32104 & 12878 & 17483 & 8474 & 6990 \\
\hline 1996 & 9797 & 11700 & 6038 & 5810 & 4463 & 4790 & 3692 & 3290 & 36749 & 47578 & 26113 & 23262 & 14439 & 18030 & 9041 & 8291 \\
\hline 1997 & 7001 & 9140 & 5553 & 6520 & 3653 & 3530 & 3401 & 2770 & 24710 & 33762 & 19058 & 25115 & 12393 & 10400 & 8294 & 6250 \\
\hline 1998 & 6777 & 7330 & 4941 & 8150 & 3586 & 4760 & 2902 & 2730 & 29851 & 34567 & 22752 & 41918 & 18203 & 21440 & 10359 & 7807 \\
\hline 1999 & 8043 & 6930 & 6262 & 6220 & 3961 & 3430 & 2829 & 2150 & 36152 & 35830 & 26154 & 34465 & 17061 & 14497 & 9966 & 5503 \\
\hline 2000 & 5037 & 5080 & 4519 & 5980 & 3040 & 3090 & 2705 & 2220 & 18331 & 21279 & 14899 & 26722 & 9148 & 10907 & 7049 & 5731 \\
\hline 2001 & 6556 & 8080 & 5494 & 4720 & 3517 & 3370 & 3269 & 2690 & 24009 & 35089 & 18391 & 19025 & 10516 & 10963 & 7585 & 6419 \\
\hline 2002 & 8433 & 9680 & 5380 & 3940 & 4075 & 2870 & 3128 & 2700 & 30172 & 34742 & 22416 & 14215 & 14220 & 8057 & 8962 & 5683 \\
\hline 2003 & 8552 & 8800 & 5918 & 5370 & 4108 & 4260 & 3272 & 3760 & 29374 & 30068 & 21590 & 19023 & 11594 & 12916 & 7996 & 8610 \\
\hline 2004 & 8394 & 9440 & 6616 & 7750 & 4062 & 3870 & 3053 & 4090 & 30481 & 34348 & 22212 & 28843 & 11611 & 12758 & 8002 & 11012 \\
\hline 2005 & 4412 & 4400 & 4324 & 3540 & 2835 & 2270 & 2904 & 2940 & 17836 & 20465 & 14783 & 15242 & 10458 & 5964 & 7563 & 8525 \\
\hline 2006 & 4285 & 3650 & 3887 & 2770 & 2793 & 2140 & 2601 & 2270 & 19677 & 17509 & 16116 & 11569 & 12328 & 6941 & 8270 & 6518 \\
\hline 2007 & 7123 & 4880 & 5863 & 5830 & 3689 & 3620 & 3205 & 3530 & 27414 & 17792 & 20574 & 18662 & 12206 & 9270 & 8225 & 7238 \\
\hline 2008 & 11507 & 9760 & 7449 & 8550 & 4937 & 5010 & 3460 & 5560 & 42257 & 34315 & 29513 & 27353 & 17005 & 15002 & 9946 & 13849 \\
\hline 2009 & 9380 & 11200 & 5754 & 5670 & 4347 & 4800 & 3232 & 3910 & 33557 & 32794 & 24497 & 18261 & 15529 & 13244 & 9429 & 9351 \\
\hline 2010 & 9360 & 8380 & 6209 & 7240 & 4339 & 5640 & 3325 & 3940 & 36202 & 29711 & 25764 & 22795 & 14119 & 15261 & 8926 & 9224 \\
\hline 2011 & 14625 & 12700 & 8231 & 7390 & 5770 & 4610 & 3474 & 3670 & 55333 & 48184 & 37284 & 32661 & 21790 & 18430 & 11565 & 12412 \\
\hline 2012 & 4256 & 2790 & 4230 & 2130 & 2783 & 1800 & 2303 & 1870 & 15399 & 10608 & 13139 & 8026 & 9070 & 6259 & 7018 & 5664 \\
\hline 2013 & 10871 & 9810 & 7915 & 7710 & 4762 & 4550 & 3486 & 2730 & 44454 & 40902 & 30744 & 33015 & 17231 & 16925 & 10024 & 7293 \\
\hline 2014 & 6012 & 7090 & 4552 & 6010 & 3350 & 3550 & 3821 & 3940 & 23275 & 29576 & 18193 & 25791 & 12111 & 12324 & 8189 & 11632 \\
\hline 2015 & 13207 & 10500 & 9104 & 12700 & 5393 & 8150 & 3180 & 2820 & 42856 & 30497 & 28995 & 33812 & 11672 & 20916 & 8025 & 5934 \\
\hline 2016 & 7966 & 7280 & 6164 & 4790 & 3939 & 5070 & 2682 & 4280 & 25352 & 21761 & 19241 & 17377 & 11080 & 18220 & 7801 & 13529 \\
\hline
\end{tabular}


Table S3.2: Predicted by the regressions (pre) and observed (obs) monthly Mississippi River discharge and nitrogen loading

\begin{tabular}{|c|c|c|c|c|c|c|c|c|c|c|c|c|c|c|c|c|}
\hline \multirow{4}{*}{ Year } & \multicolumn{16}{|c|}{ Mississippi River } \\
\hline & \multicolumn{8}{|c|}{ Discharge $\left(\mathrm{m}^{3} / \mathrm{s}\right)$} & \multicolumn{8}{|c|}{ Loading (t/mo) } \\
\hline & \multicolumn{2}{|c|}{ Jun } & \multicolumn{2}{|c|}{ Jul } & \multicolumn{2}{|c|}{ Aug } & \multicolumn{2}{|c|}{ Sep } & \multicolumn{2}{|c|}{ Jun } & \multicolumn{2}{|c|}{ Jul } & \multicolumn{2}{|c|}{ Aug } & \multicolumn{2}{|c|}{ Sep } \\
\hline & pre & obs & pre & obs & pre & obs & pre & obs & pre & obs & pre & obs & pre & obs & pre & obs \\
\hline 1980 & 14630 & 13600 & 11343 & 9490 & 8135 & 7860 & 8274 & 8010 & 63302 & 65152 & 47753 & 41706 & 26594 & 28307 & 20013 & 31300 \\
\hline 1981 & 17920 & 20600 & 14115 & 14900 & 9121 & 11400 & 6541 & 8170 & 85438 & 101948 & 64589 & 84155 & 32983 & 62810 & 25417 & 34298 \\
\hline 1982 & 19984 & 20400 & 15303 & 14600 & 9725 & 9990 & 9036 & 9200 & 111437 & 143000 & 81310 & 92394 & 41684 & 53563 & 26813 & 42533 \\
\hline 1983 & 30025 & 34300 & 18650 & 14300 & 12537 & 8250 & 7422 & 6450 & 158722 & 139385 & 108588 & 78336 & 58501 & 34090 & 25718 & 20451 \\
\hline 1984 & 20899 & 23300 & 13811 & 14000 & 10012 & 8710 & 7839 & 6850 & 98964 & 123543 & 69521 & 92867 & 39435 & 34138 & 20464 & 19808 \\
\hline 1985 & 13430 & 13100 & 10561 & 9260 & 7762 & 7550 & 8034 & 7330 & 63122 & 57531 & 46965 & 32609 & 26875 & 19074 & 18873 & 16327 \\
\hline 1986 & 14582 & 17700 & 11759 & 11800 & 8115 & 7530 & 6669 & 6360 & 80192 & 112690 & 59026 & 63614 & 32276 & 28958 & 21600 & 16855 \\
\hline 1987 & 14336 & 10900 & 11802 & 9360 & 8037 & 5870 & 6600 & 5690 & 69577 & 55102 & 52856 & 34909 & 28267 & 14390 & 22124 & 13905 \\
\hline 1988 & 7504 & 4990 & 7294 & 3600 & 5765 & 3790 & 8186 & 3720 & 28529 & 13023 & 23687 & 5649 & 14430 & 5606 & 15706 & 4847 \\
\hline 1989 & 15744 & 19200 & 12198 & 19900 & 8475 & 8400 & 7990 & 9310 & 60823 & 60366 & 47098 & 48845 & 25237 & 15385 & 21538 & 19664 \\
\hline 1990 & 25018 & 31400 & 17121 & 16000 & 11160 & 10100 & 6481 & 8560 & 123552 & 152546 & 88420 & 107462 & 45988 & 50702 & 26560 & 34340 \\
\hline 1991 & 26414 & 20800 & 17243 & 10500 & 11552 & 6670 & 6937 & 6260 & 137626 & 126306 & 95863 & 58028 & 51343 & 24382 & 25282 & 16435 \\
\hline 1992 & 8525 & 11500 & 7723 & 11200 & 6133 & 13700 & 6285 & 8200 & 45027 & 45566 & 34027 & 48241 & 20980 & 73102 & 15673 & 27006 \\
\hline 1993 & 22938 & 21400 & 14949 & 19300 & 10591 & 22600 & 8664 & 15300 & 115379 & 112194 & 80188 & 118201 & 44809 & 129349 & 21813 & 83362 \\
\hline 1994 & 15222 & 12100 & 10543 & 11000 & 8331 & 8130 & 8206 & 6550 & 52488 & 42928 & 39023 & 37795 & 23629 & 20355 & 16420 & 12330 \\
\hline 1995 & 28816 & 31100 & 19949 & 17700 & 12190 & 11200 & 7552 & 6980 & 148559 & 119520 & 106812 & 73396 & 52928 & 39035 & 31448 & 16402 \\
\hline 1996 & 22845 & 27800 & 15901 & 13800 & 10553 & 11200 & 8783 & 7580 & 108282 & 120410 & 78280 & 71102 & 41135 & 49456 & 25121 & 20769 \\
\hline 1997 & 16390 & 21300 & 12093 & 15200 & 8676 & 8270 & 8127 & 6530 & 71260 & 85186 & 52775 & 60286 & 29443 & 24918 & 20274 & 15357 \\
\hline 1998 & 15885 & 17100 & 11094 & 19100 & 8532 & 11100 & 7001 & 6390 & 70789 & 81083 & 50718 & 95731 & 30188 & 45490 & 17465 & 14843 \\
\hline 1999 & 18721 & 16100 & 13442 & 14600 & 9369 & 8040 & 6836 & 5000 & 106351 & 104707 & 74928 & 88893 & 41555 & 36572 & 21925 & 12957 \\
\hline 2000 & 11823 & 11900 & 10204 & 14100 & 7240 & 7320 & 6555 & 5110 & 47522 & 57404 & 37897 & 78568 & 20730 & 26753 & 19944 & 12637 \\
\hline 2001 & 15158 & 19200 & 12123 & 11000 & 8293 & 7870 & 7829 & 6270 & 85153 & 121619 & 62349 & 63865 & 33905 & 27439 & 22101 & 15801 \\
\hline 2002 & 18892 & 23700 & 13398 & 9700 & 9421 & 6720 & 7512 & 6270 & 94489 & 102801 & 67602 & 40437 & 37445 & 17896 & 21548 & 16069 \\
\hline 2003 & 19866 & 20500 & 14520 & 12500 & 9698 & 9950 & 7837 & 8770 & 85290 & 73729 & 63743 & 46583 & 33301 & 29705 & 24185 & 20424 \\
\hline 2004 & 19567 & 22100 & 14597 & 18000 & 9609 & 9040 & 7342 & 9560 & 82223 & 105046 & 62274 & 82273 & 32006 & 32300 & 24860 & 31175 \\
\hline 2005 & 10357 & 10300 & 9081 & 8310 & 6759 & 5320 & 7005 & 6700 & 44643 & 51898 & 35040 & 36541 & 20171 & 11757 & 17965 & 16424 \\
\hline 2006 & 10063 & 8520 & 8742 & 6520 & 6662 & 5030 & 6318 & 5250 & 50570 & 45046 & 38259 & 24619 & 22678 & 14307 & 17130 & 14367 \\
\hline 2007 & 16629 & 11400 & 12723 & 13600 & 8743 & 8500 & 7686 & 8240 & 96467 & 58336 & 69220 & 47943 & 37933 & 24456 & 22192 & 26493 \\
\hline 2008 & 26572 & 22900 & 17298 & 19800 & 11596 & 11900 & 8261 & 13000 & 134343 & 123993 & 93922 & 116997 & 50200 & 51038 & 25279 & 32924 \\
\hline 2009 & 21873 & 25800 & 14647 & 13200 & 10286 & 11300 & 7747 & 9310 & 93845 & 99627 & 67573 & 59025 & 37033 & 32443 & 22081 & 25662 \\
\hline 2010 & 21729 & 19500 & 15505 & 16900 & 10234 & 13100 & 7955 & 9400 & 94532 & 85273 & 70005 & 79933 & 36271 & 52511 & 25184 & 26967 \\
\hline 2011 & 33637 & 29400 & 20297 & 17300 & 13498 & 11300 & 8291 & 8640 & 130702 & 126513 & 92985 & 88569 & 48326 & 48527 & 26979 & 28920 \\
\hline 2012 & 9983 & 6480 & 8905 & 5010 & 6633 & 4270 & 5641 & 4470 & 38066 & 22103 & 30847 & 14271 & 17656 & 10947 & 17884 & 10418 \\
\hline 2013 & 25395 & 23000 & 17154 & 17800 & 11267 & 10700 & 8318 & 6520 & 133928 & 120485 & 94353 & 85009 & 49718 & 39466 & 26209 & 17208 \\
\hline 2014 & 14058 & 16500 & 11030 & 14200 & 7957 & 8340 & 9071 & 9140 & 61880 & 75217 & 46687 & 71543 & 26166 & 31228 & 19685 & 32470 \\
\hline 2015 & 29842 & 22800 & 20601 & 29000 & 12465 & 18400 & 7629 & 7450 & 138028 & 116387 & 101115 & 130076 & 49036 & 71268 & 32390 & 24406 \\
\hline 2016 & 17926 & 16200 & 13240 & 12300 & 9132 & 12400 & 6501 & 11100 & 87152 & 82778 & 63664 & 63570 & 34571 & 62689 & 22265 & 51302 \\
\hline
\end{tabular}




\section{S4 Forecast skill assessment}

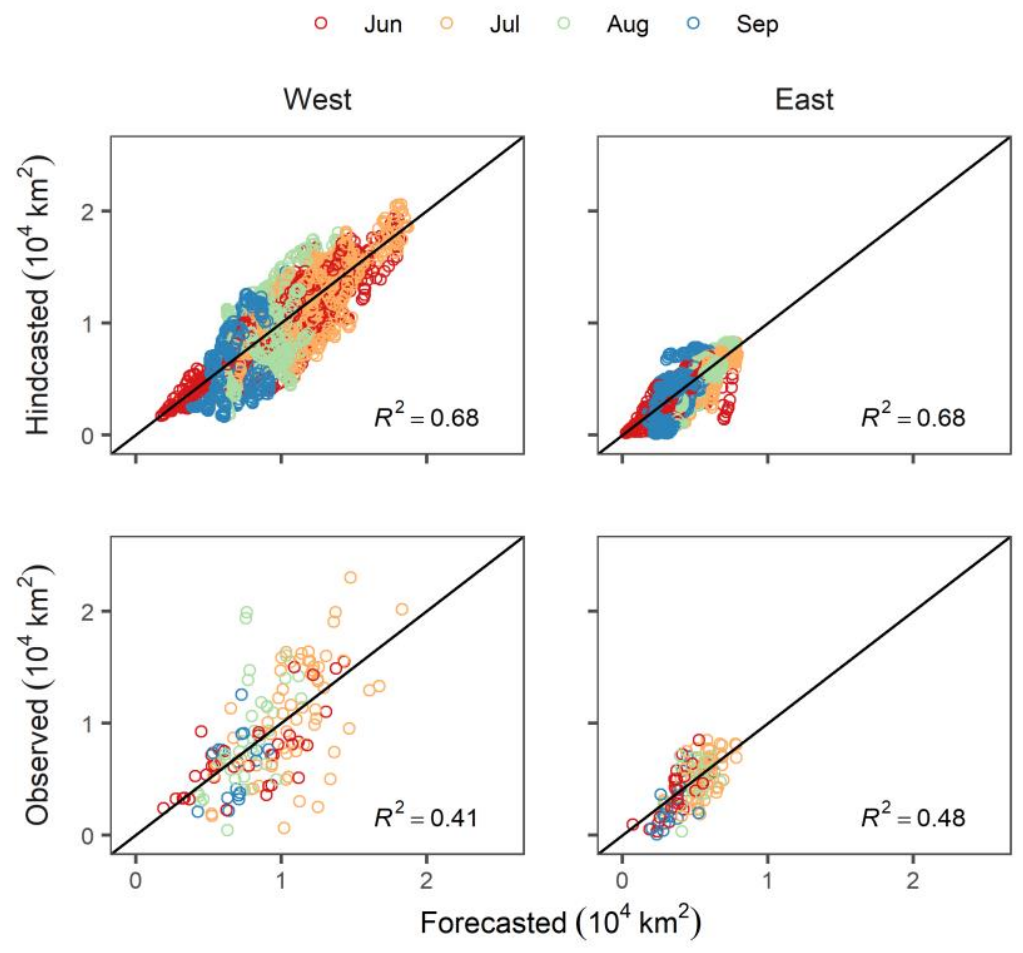

Figure S4.1: Averaged daily hindcasted and observed versus pseudo-forecasted HA for the west and east sections. Diagonal line represents perfect prediction.

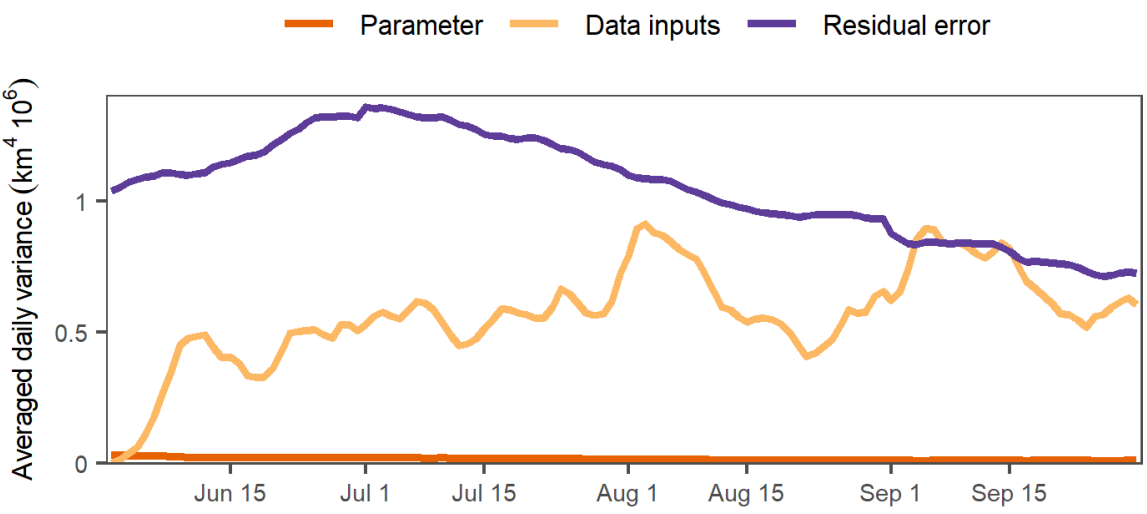

Figure S4.2: Averaged daily variance of total HA due to different sources of uncertainty. 
S5 HA pseudo-forecast for west and east sections of the shelf
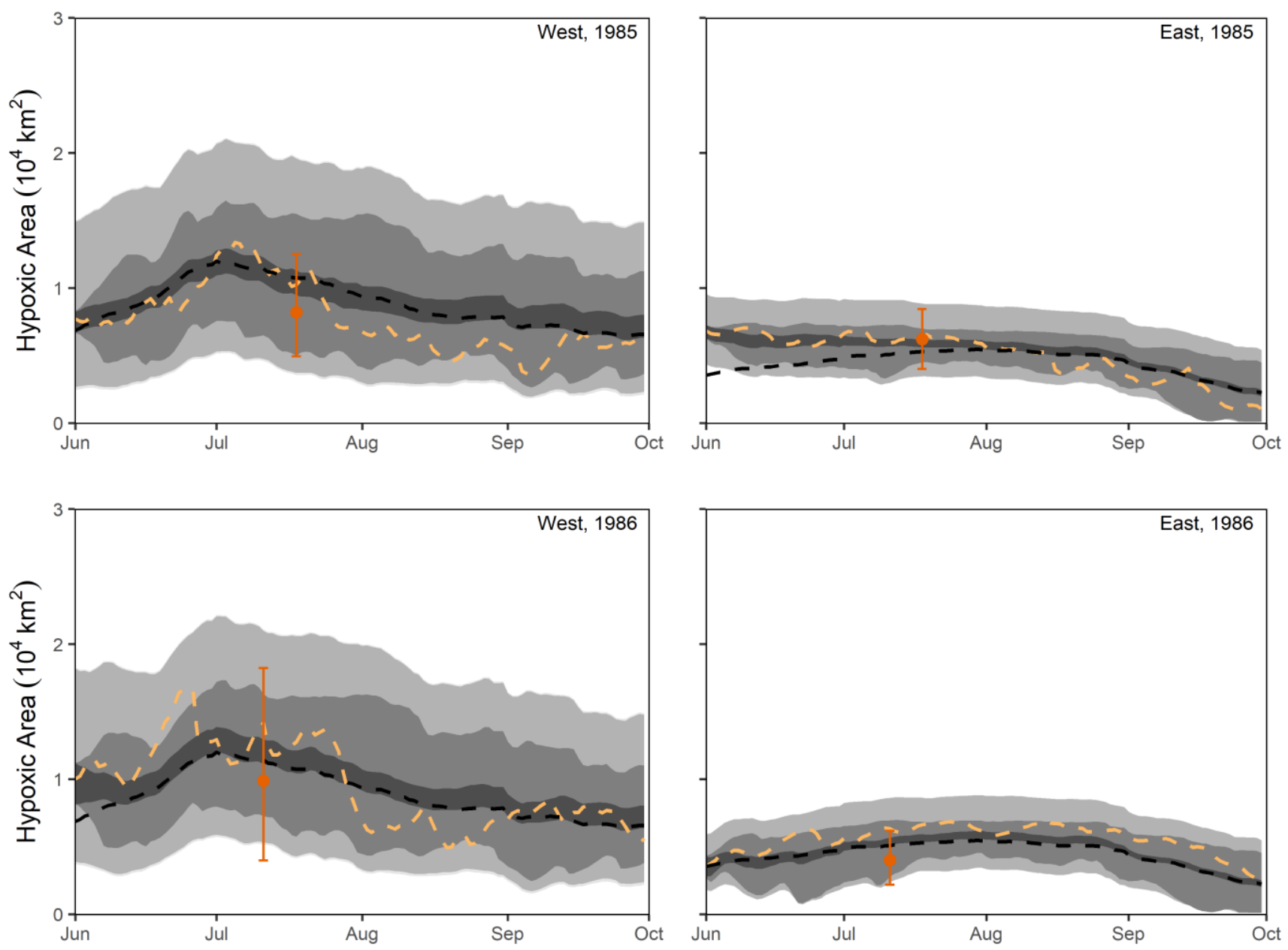

Figure S5.1: Daily pseudo-forecasts of HA for the west and east sections in 1993 (top) and 2009 (bottom), including 95\% IQR of the predictive distribution, distinguishing between i) parameter, ii) hydrometeorological inputs, iii) mechanistic model error, and iv) regression related to transformation (BWDO to HA) uncertainties (shades of gray from darkest to lightest). Yellow dashed line is hindcasted estimate, black dashed line is the 32 year average hindcast, orange points and error bars represent the mean and associated $95 \%$ confidence interval of the (geostatistically estimated) hypoxia observations. 

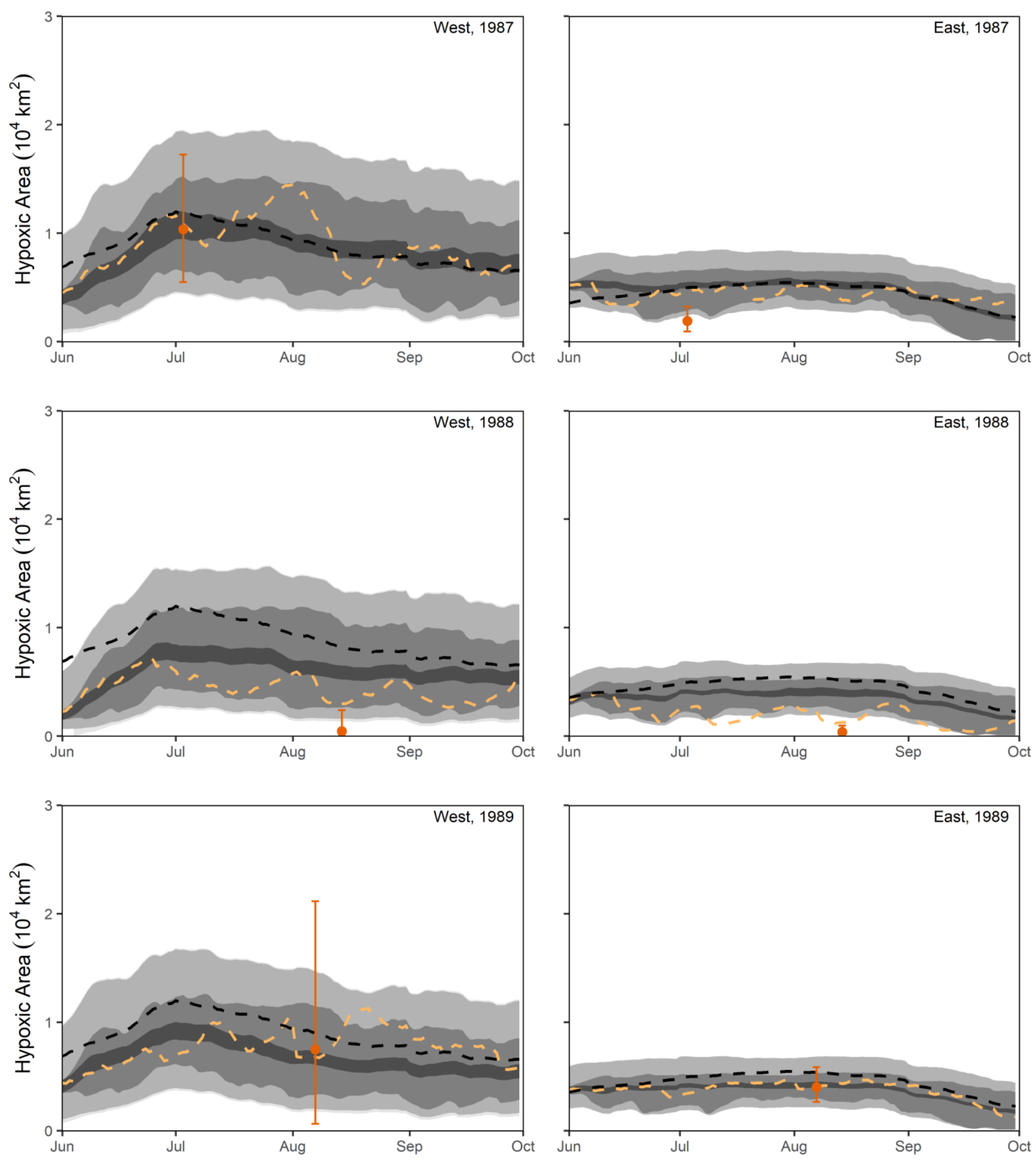

Figure S5.2: Pseudo-forecast as in Fig. S5.1 but for different years. 

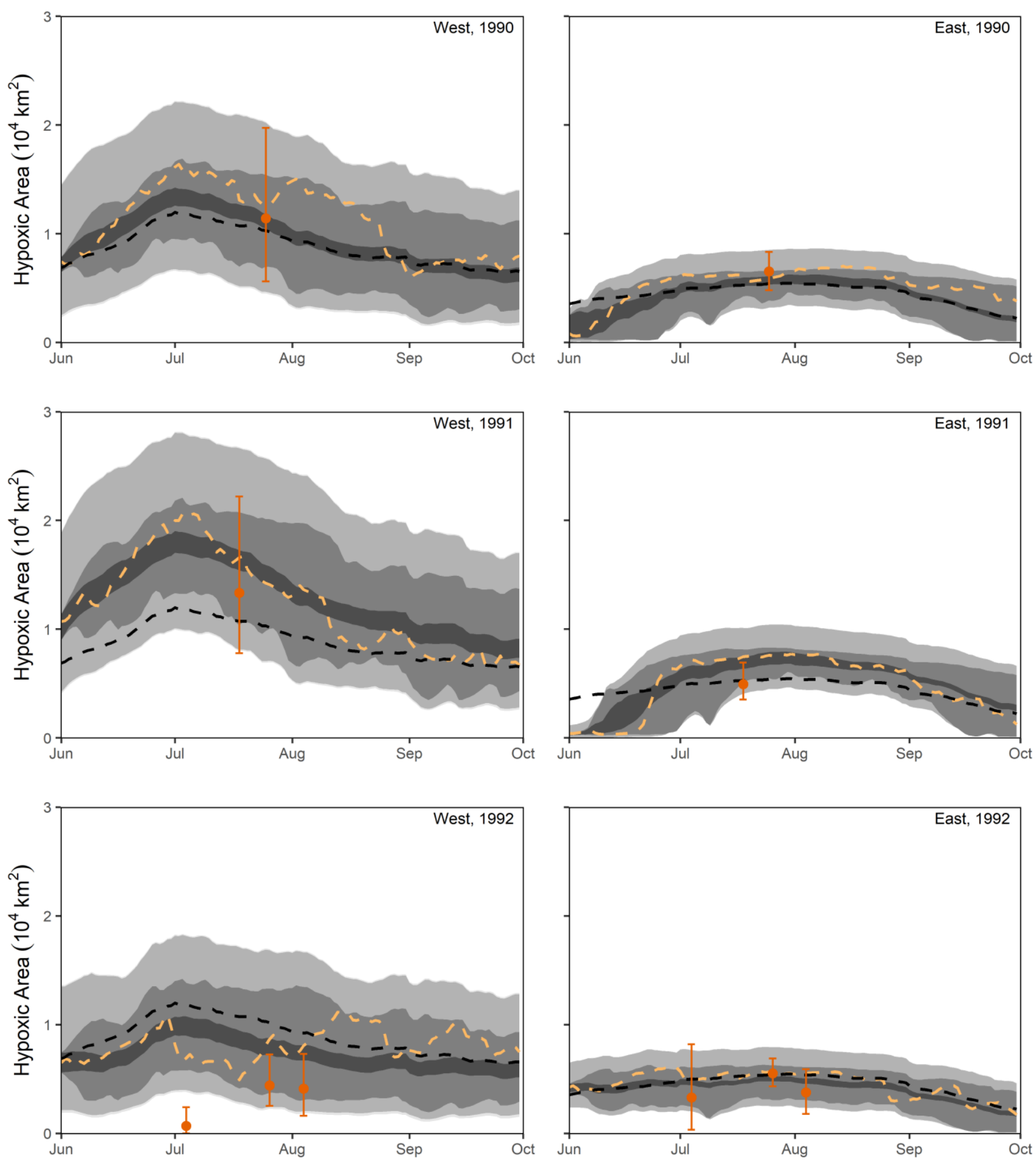

Figure S5.3: Pseudo-forecast as in Fig. S5.1 but for different years. 

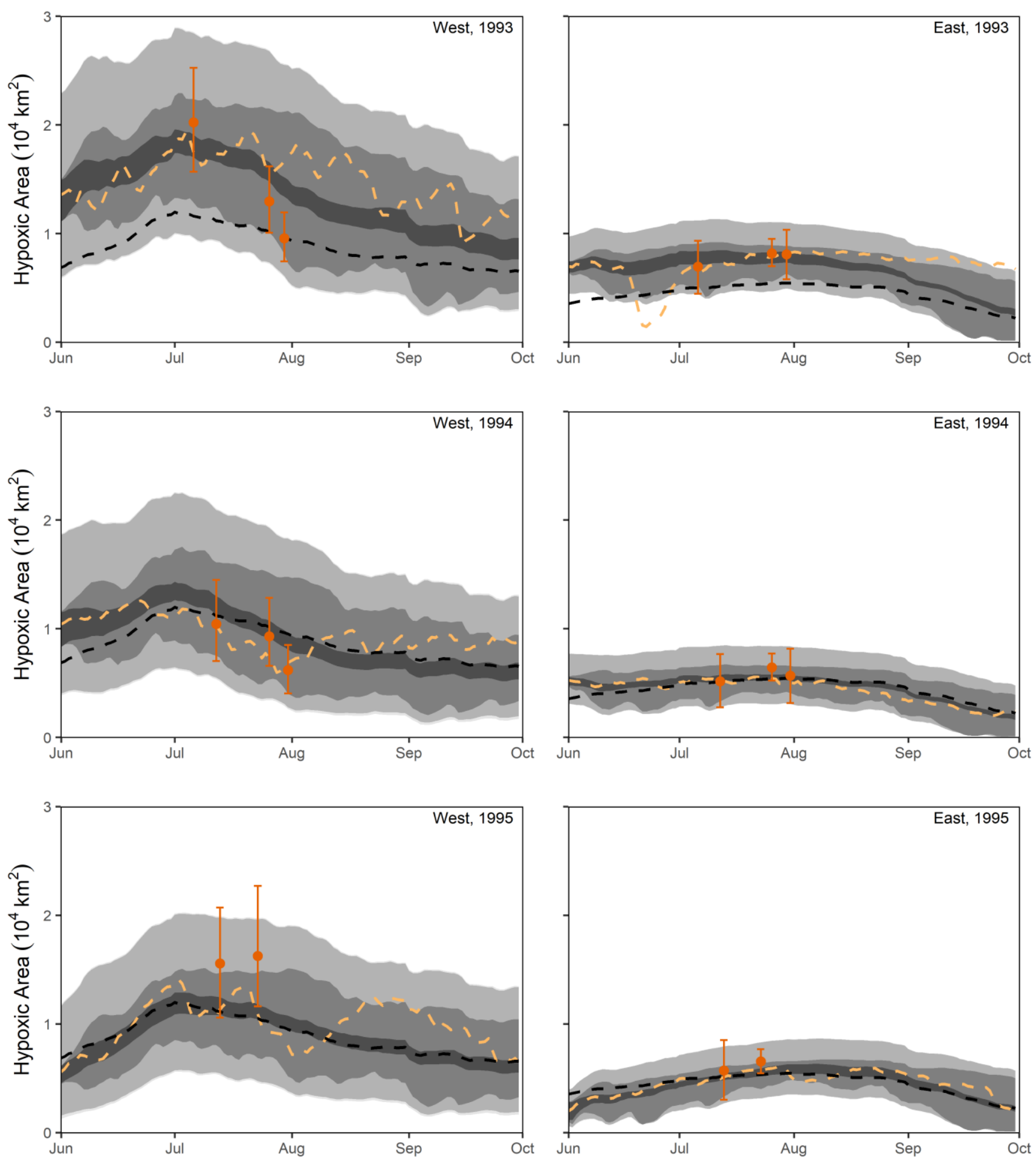

Figure S5.4: Pseudo-forecast as in Fig. S5.1 but for different years. 

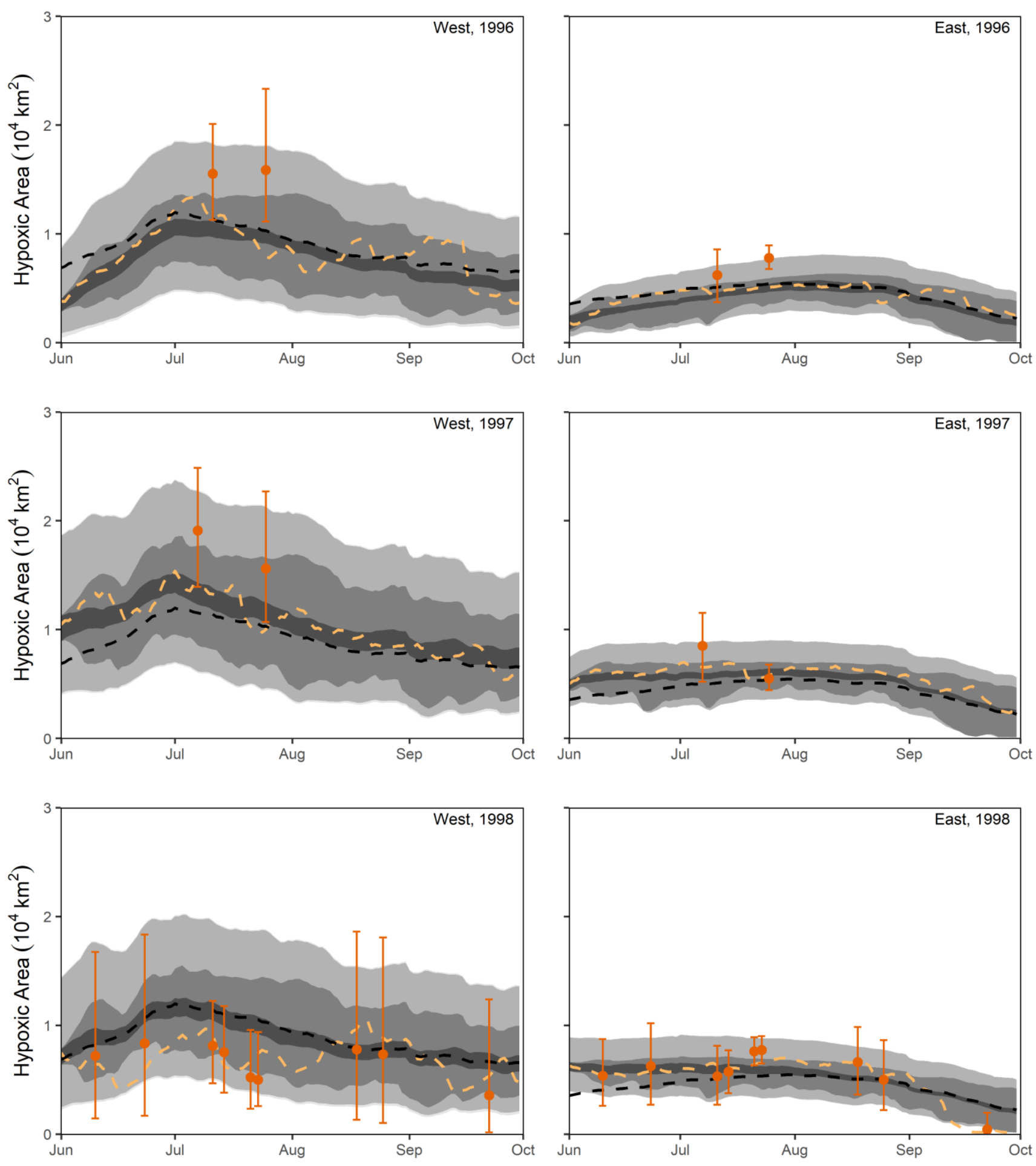

Figure S5.5: Pseudo-forecast as in Fig. S5.1 but for different years. 

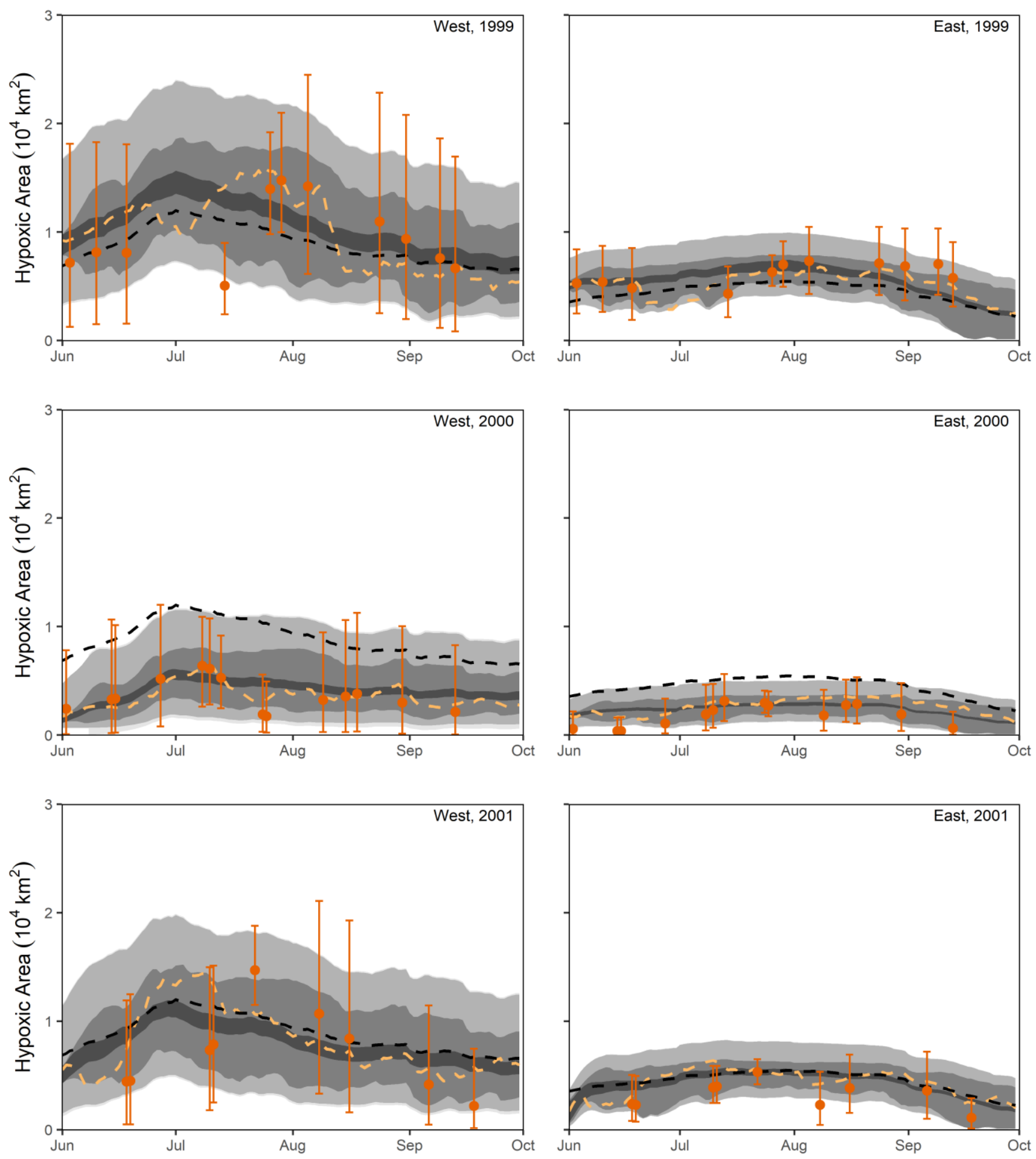

Figure S5.6: Pseudo-forecast as in Fig. S5.1 but for different years. 

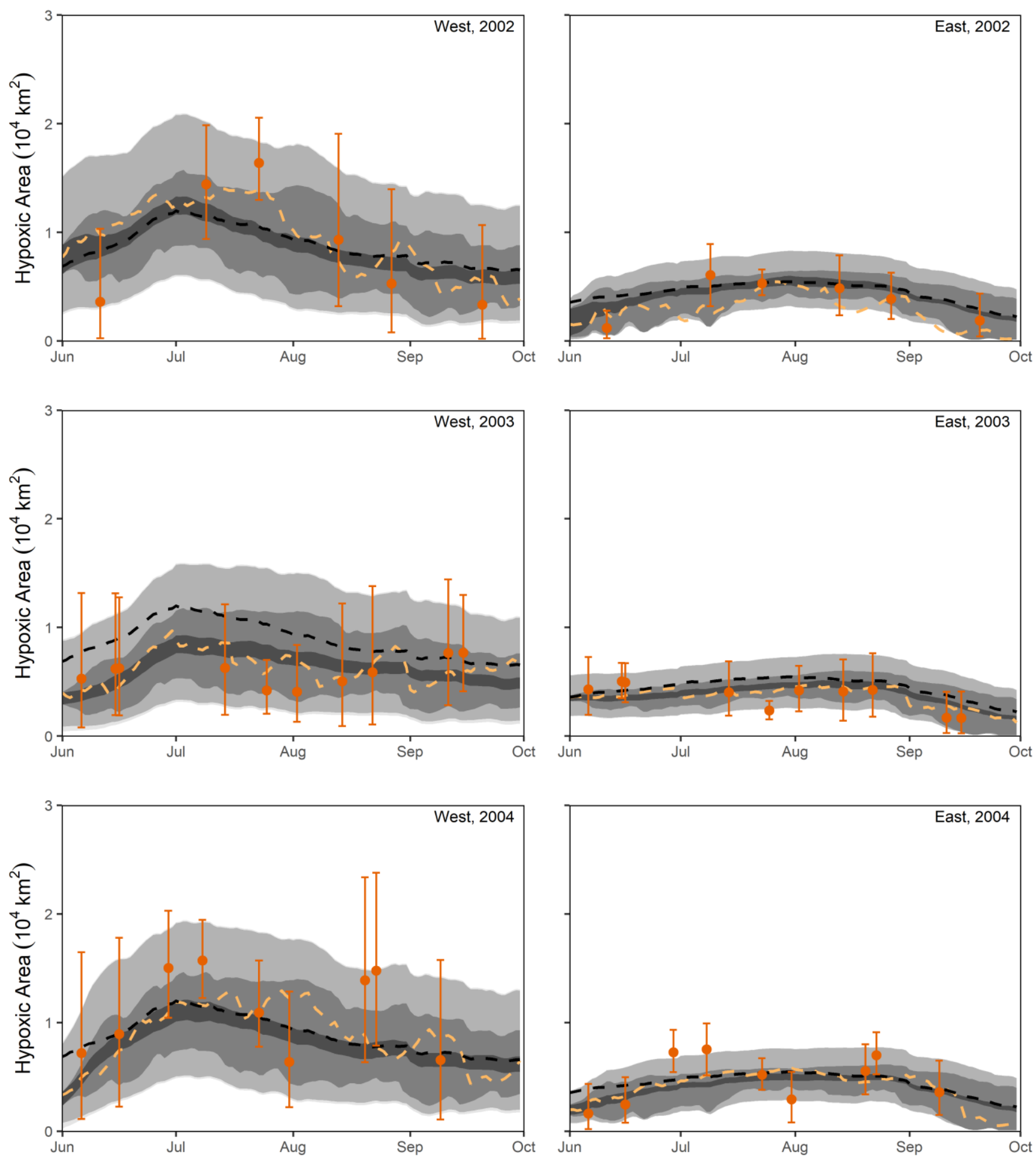

Figure S5.7: Pseudo-forecast as in Fig. S5.1 but for different years. 

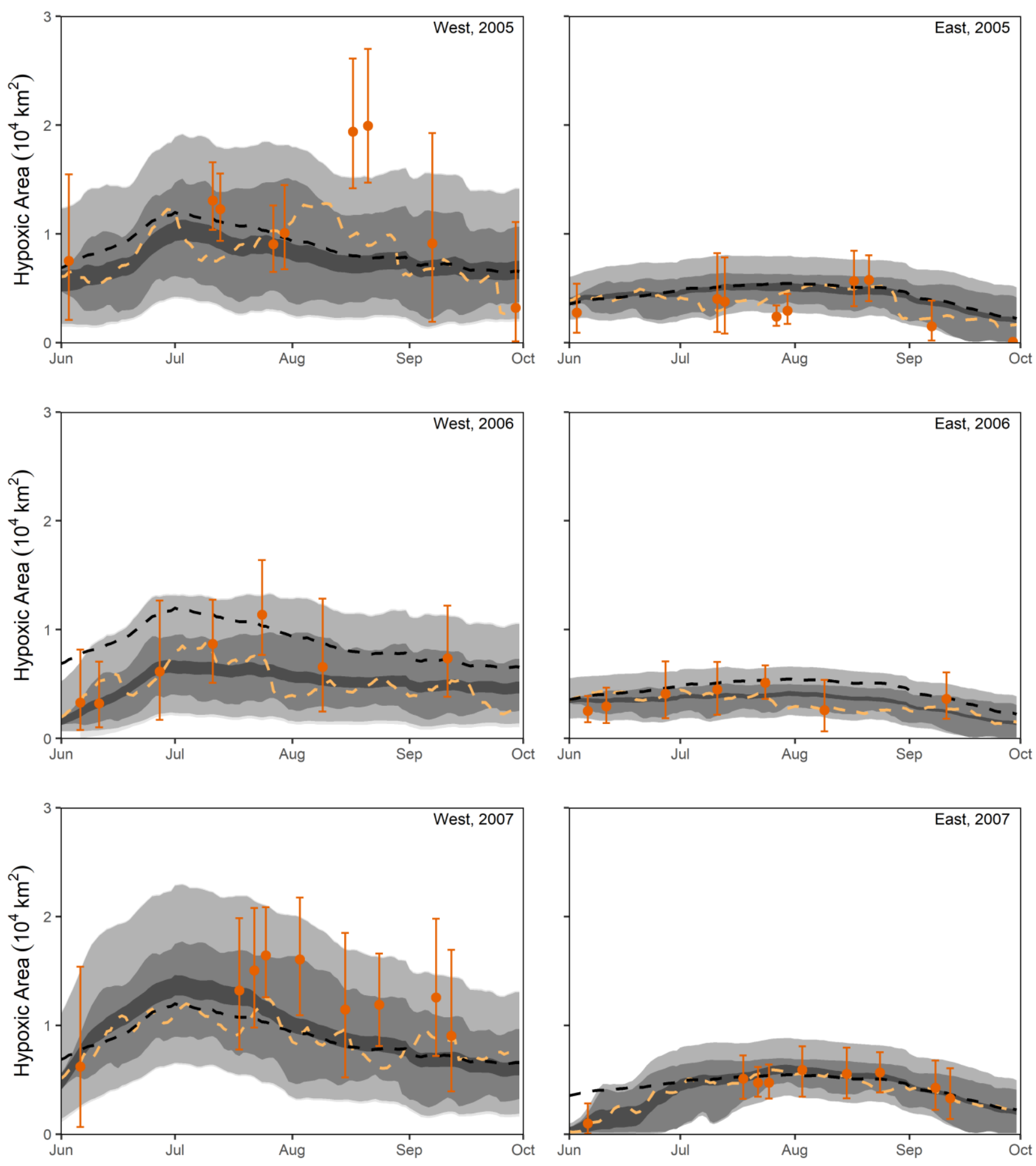

Figure S5.8: Pseudo-forecast as in Fig. S5.1 but for different years. 

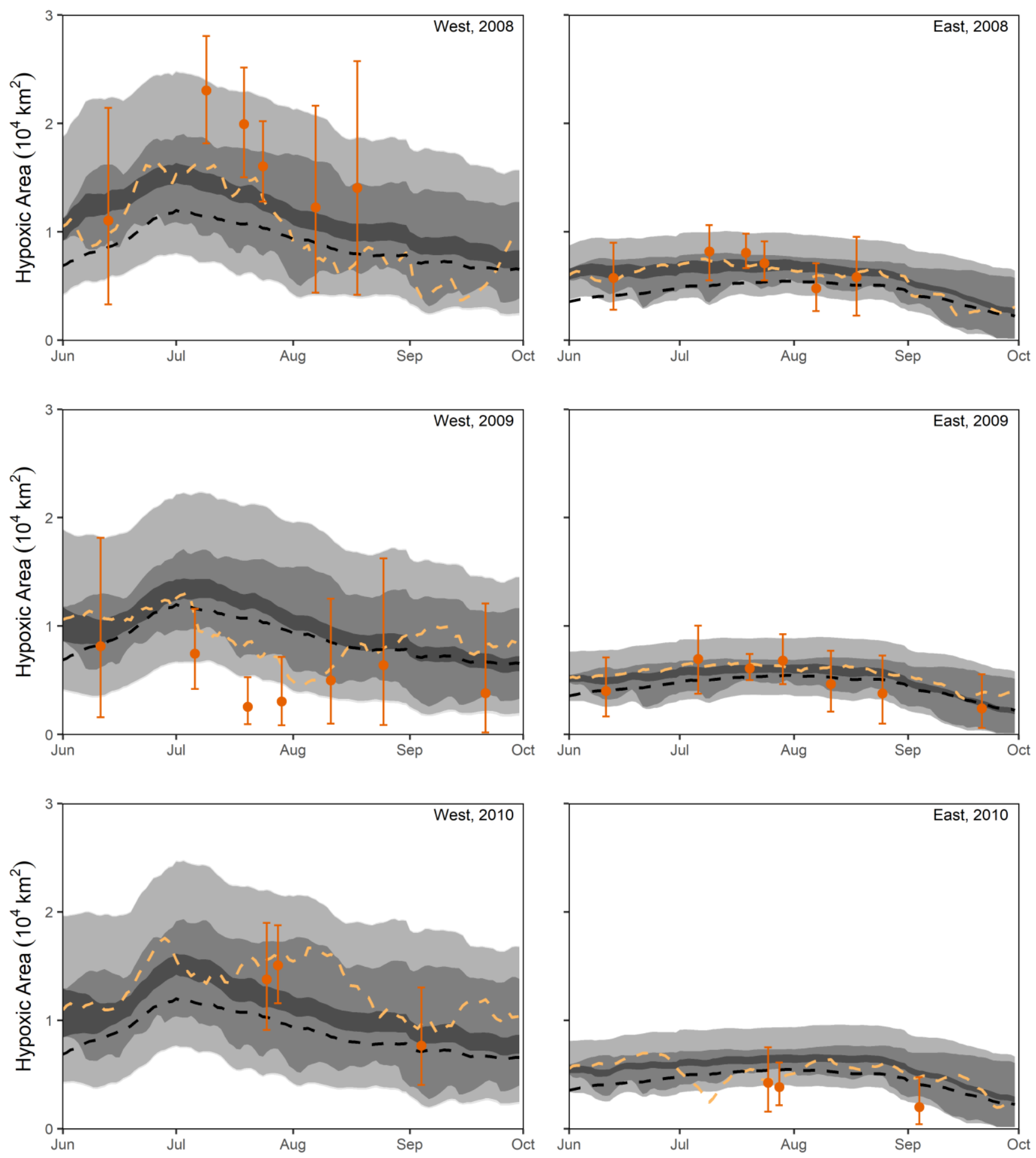

Figure S5.9: Pseudo-forecast as in Fig. S5.1 but for different years. 

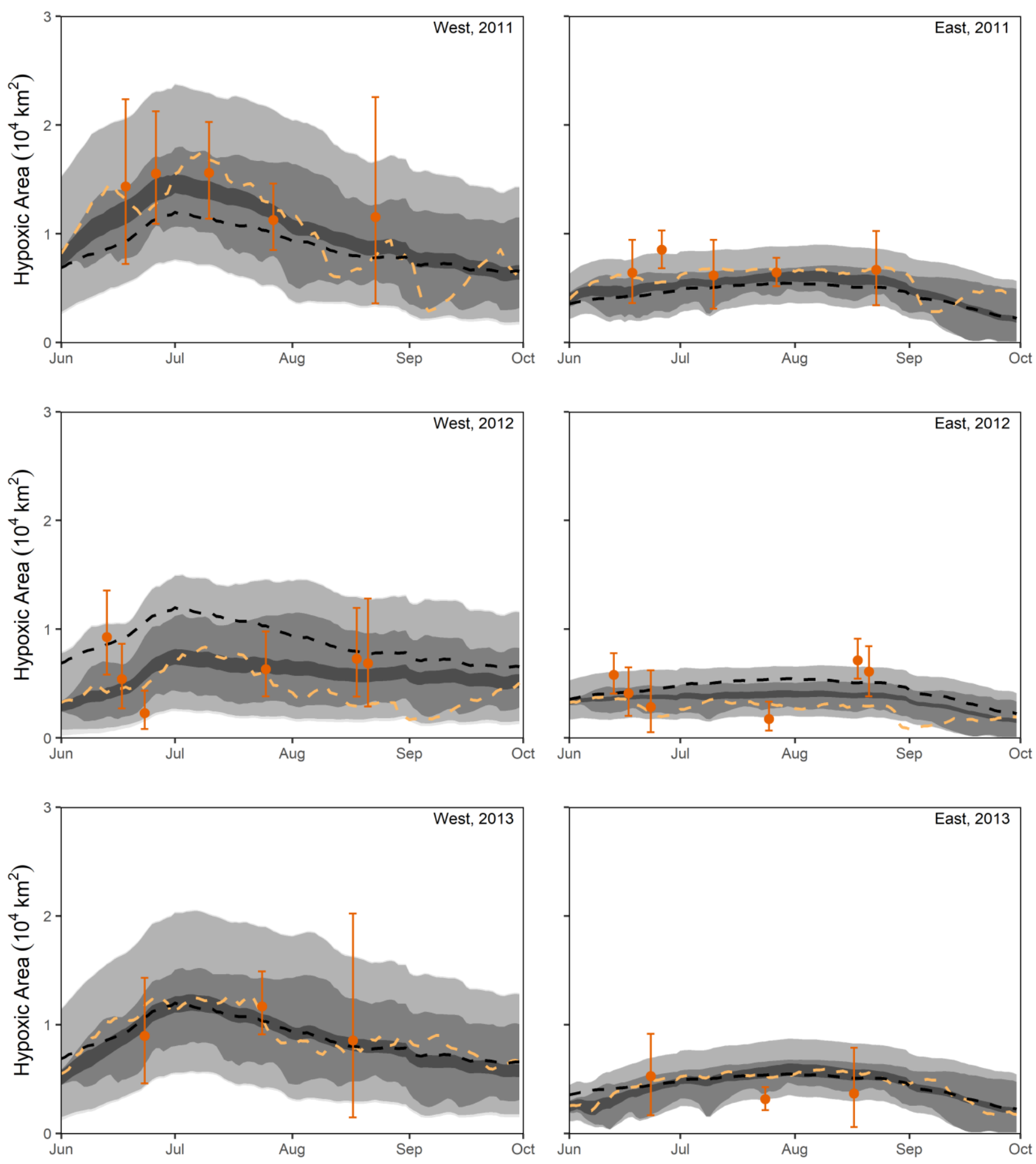

Figure S5.10: Pseudo-forecast as in Fig. S5.1 but for different years. 

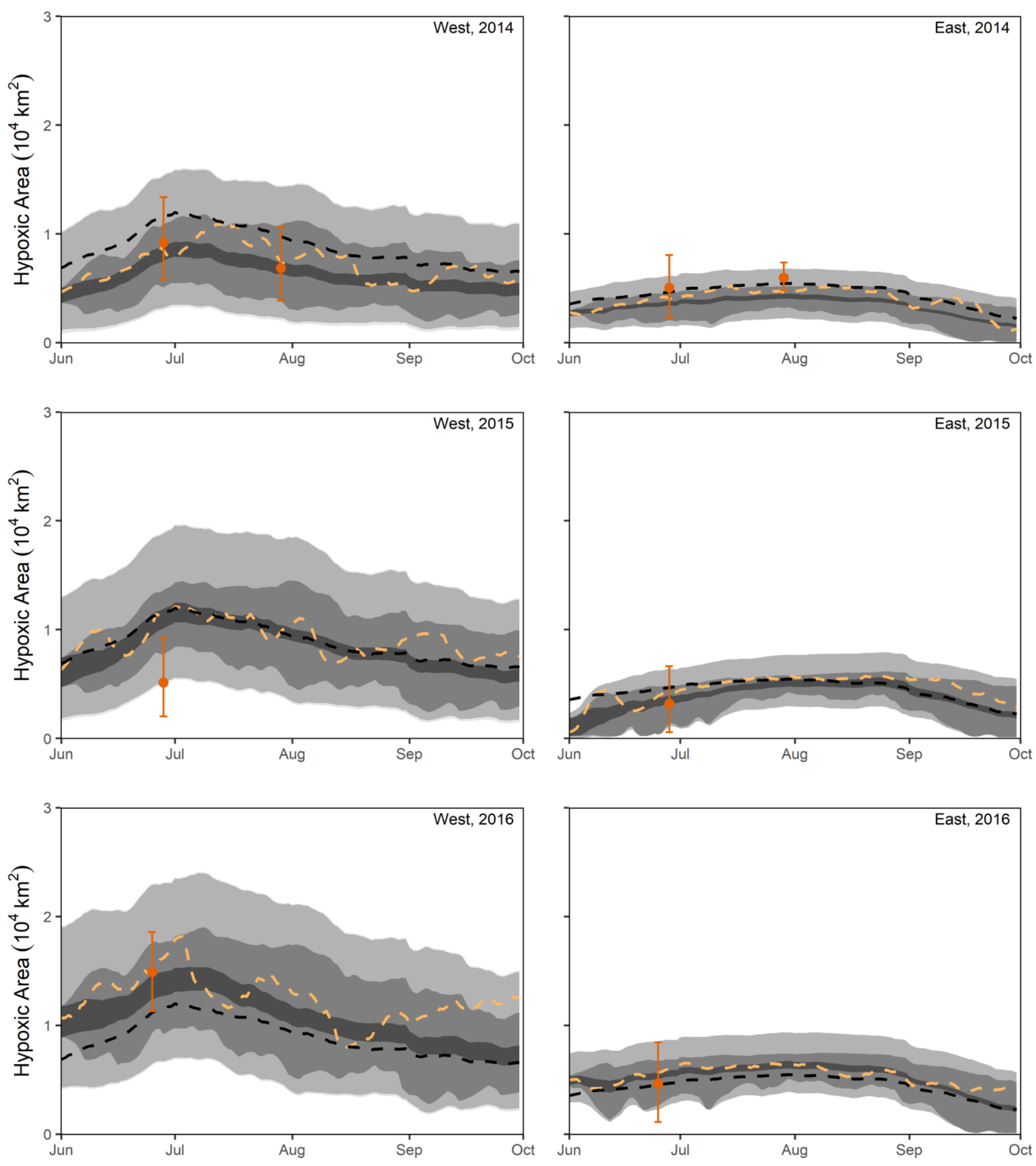

Figure S5.11: Pseudo-forecast as in Fig. S5.1 but for different years. 

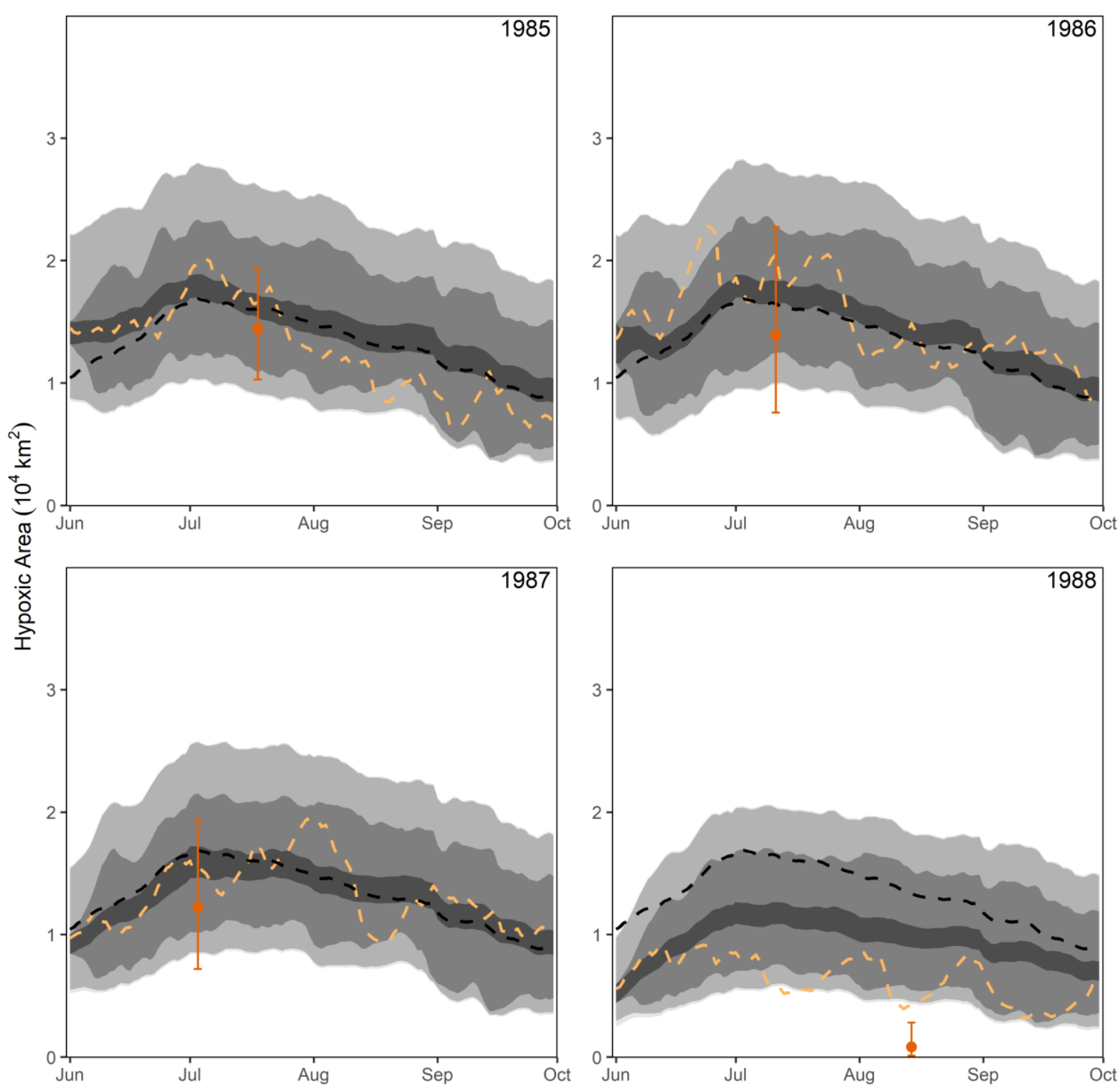

Figure S6.1: Daily pseudo-forecasts of total HA, including 95\% IQR of the predictive distribution, distinguishing between i) parameter, ii) hydrometeorological inputs, iii) mechanistic model error and iv) regression related to transformation (BWDO to HA) uncertainties (shades of gray from darkest to lightest). Yellow dashed line is hindcasted estimate, black dashed line is the 32 year average hindcast, orange points and error bars represent the mean and associated $95 \%$ confidence interval of the (geostatistically estimated) hypoxia observations. 

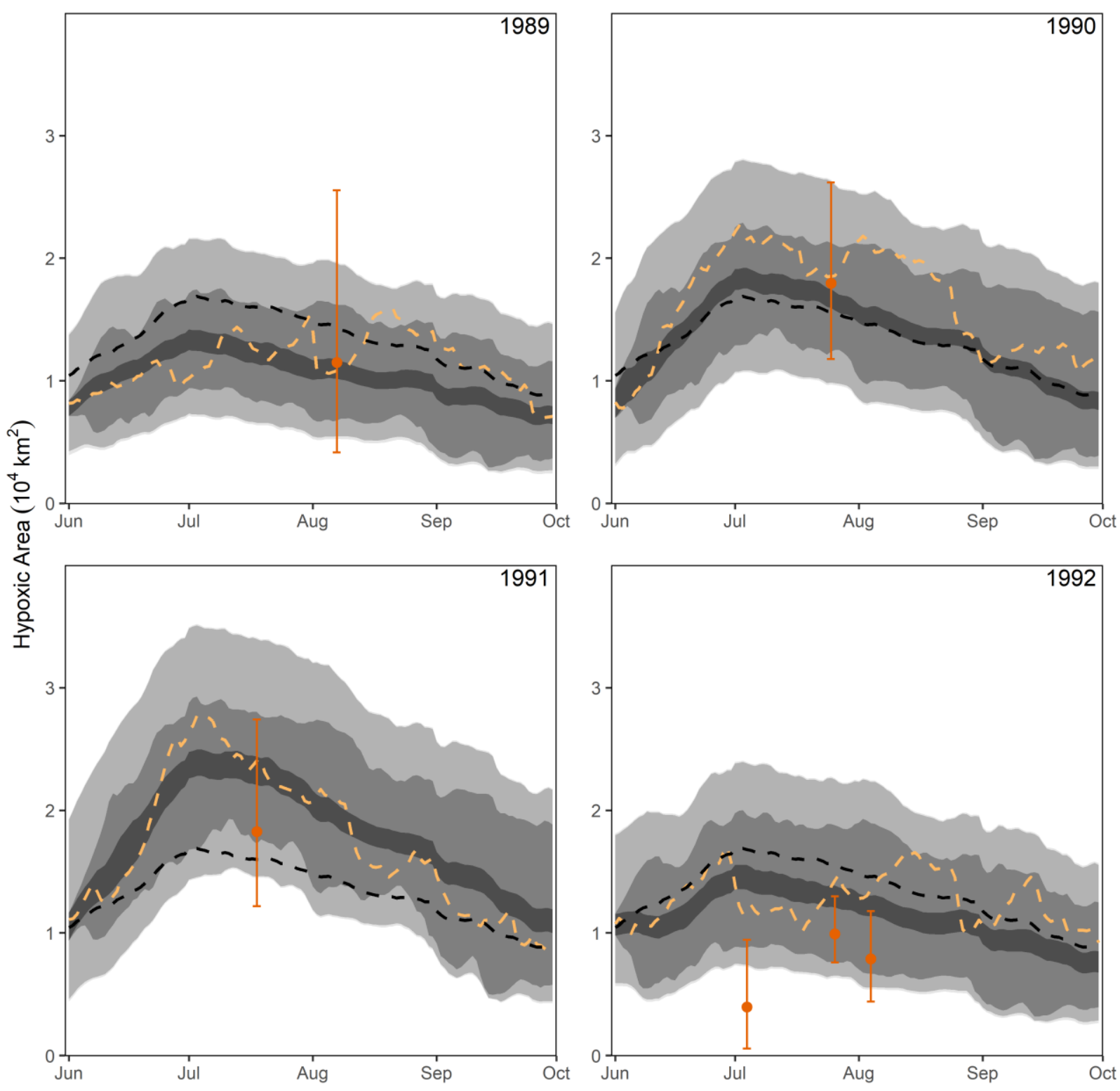

Figure S6.2: Pseudo-forecast as in Fig. S6.1 but for different years. 

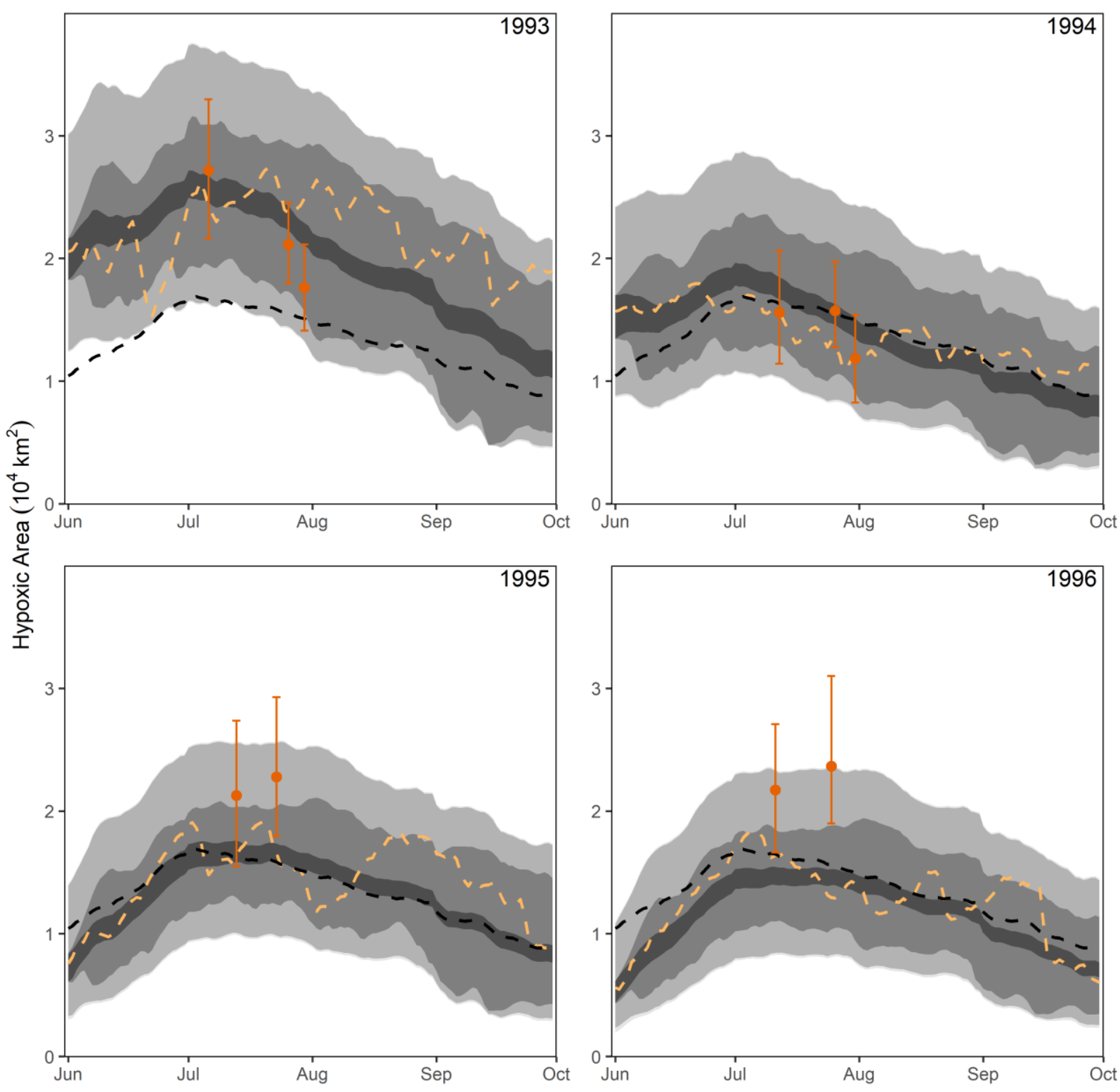

Figure S6.3: Pseudo-forecast as in Fig. S6.1 but for different years. 

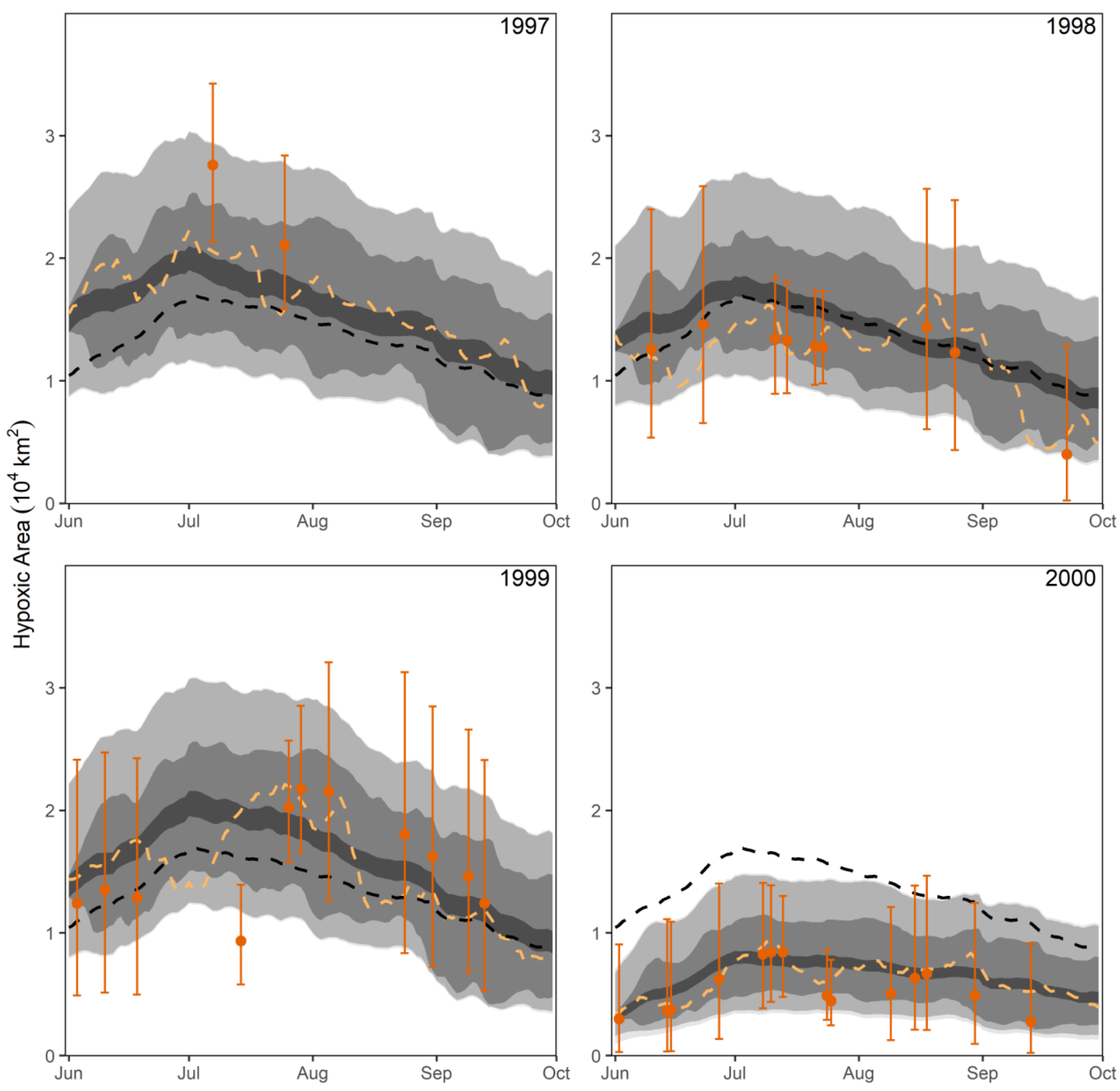

Figure S6.4: Pseudo-forecast as in Fig. S6.1 but for different years. 

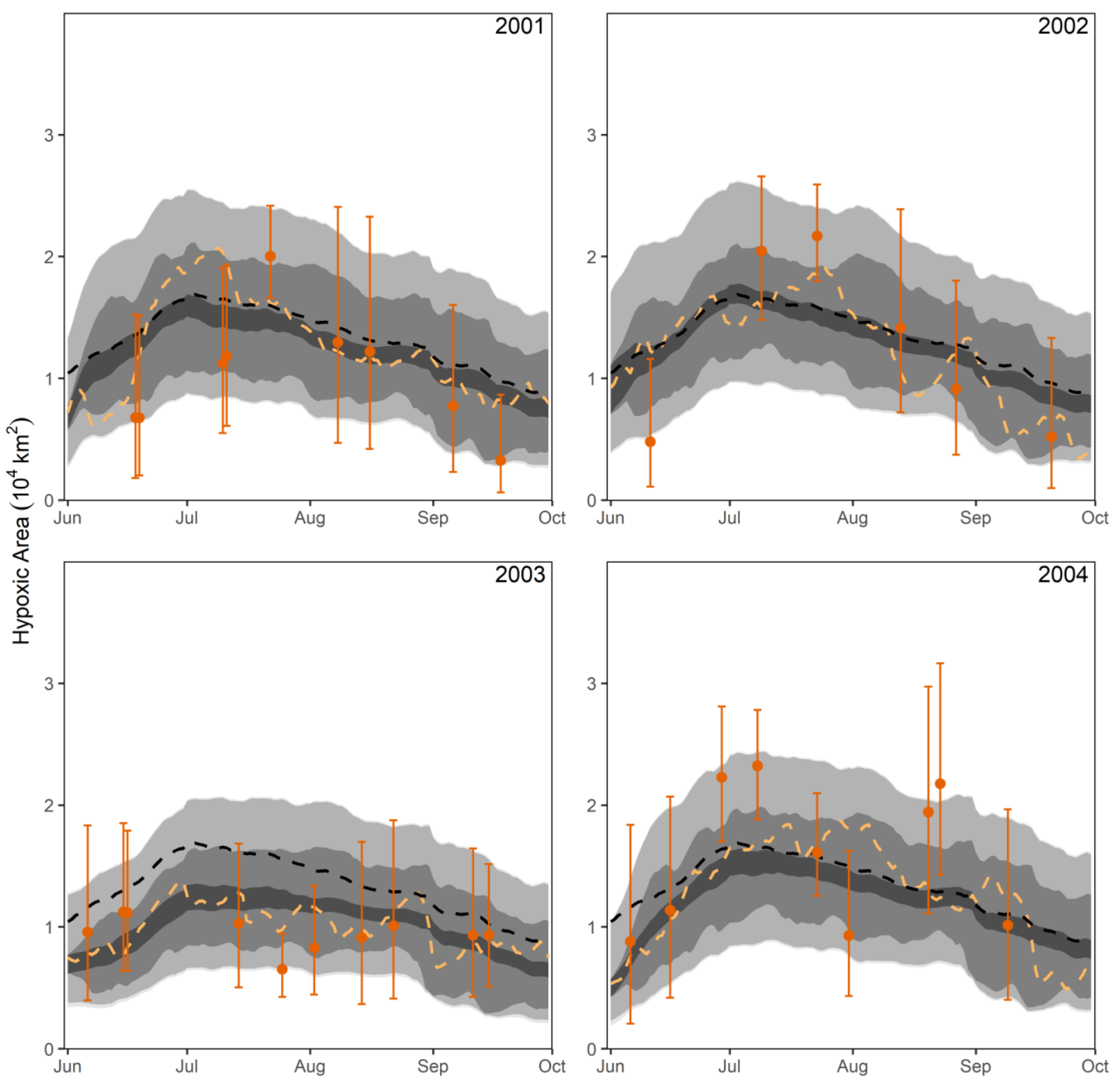

Figure S6.5: Pseudo-forecast as in Fig. S6.1 but for different years. 

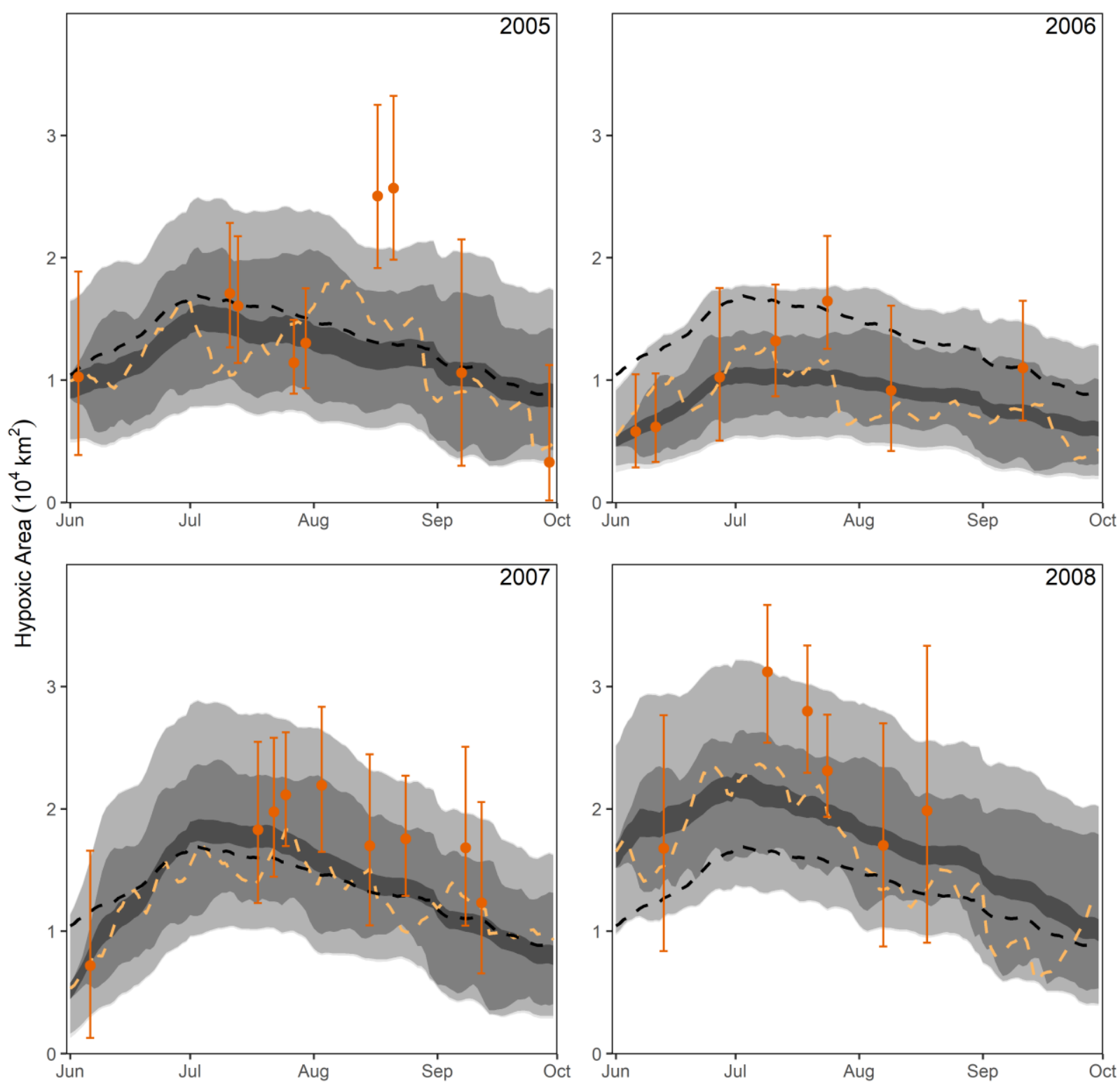

Figure S6.6: Pseudo-forecast as in Fig. S6.1 but for different years. 

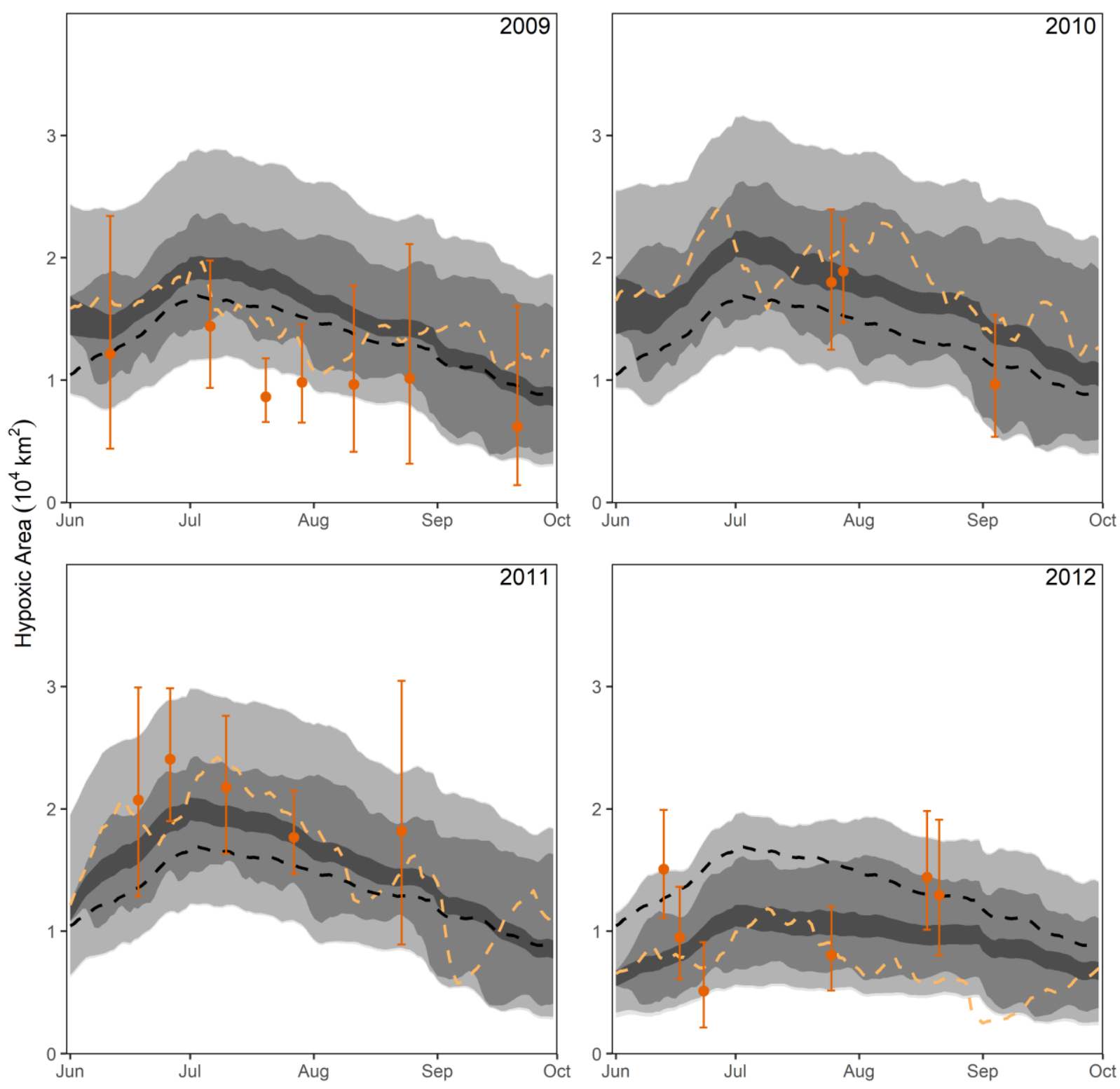

Figure S6.7: Pseudo-forecast as in Fig. S6.1 but for different years. 

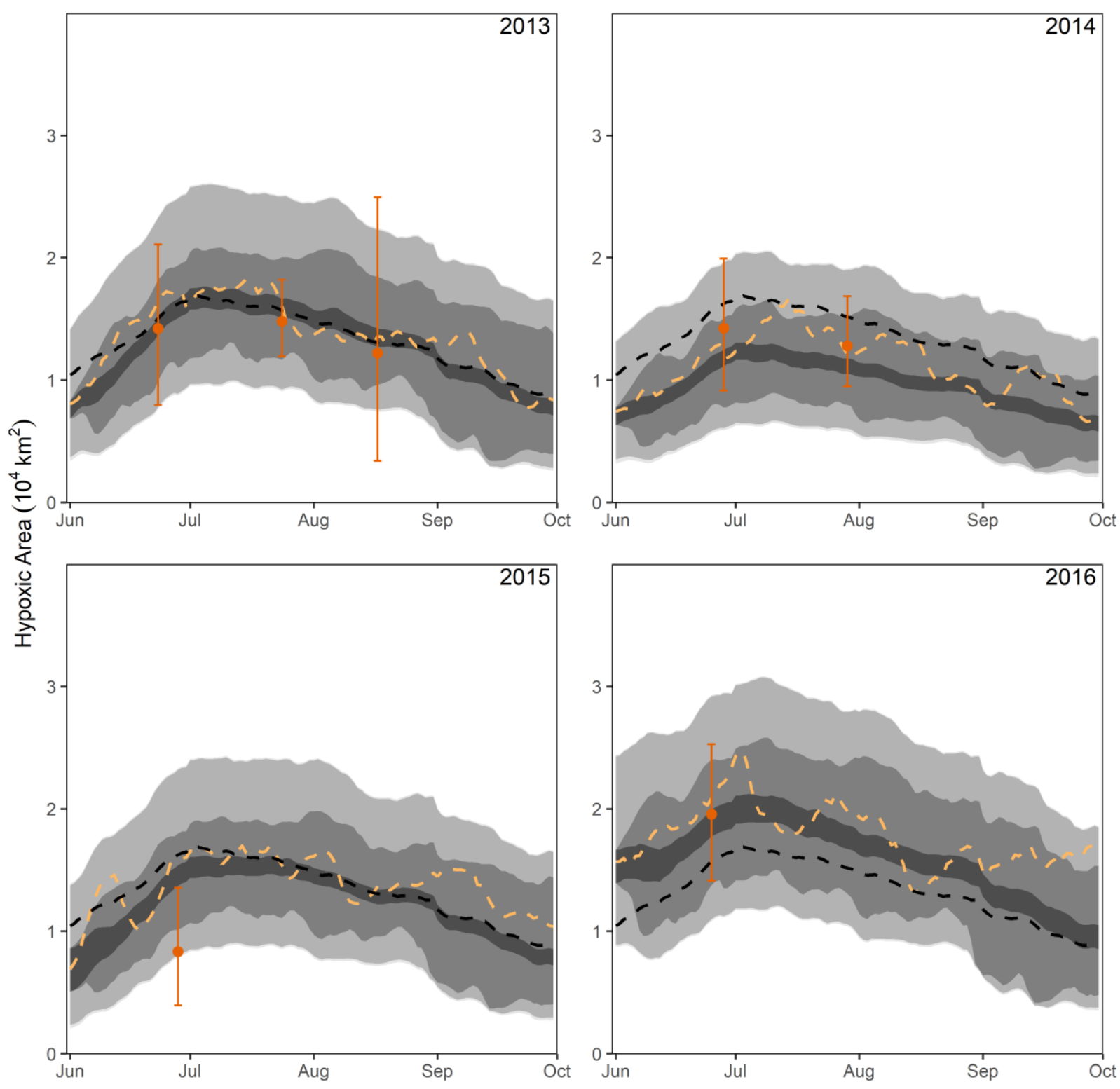

Figure S6.8: Pseudo-forecast as in Fig. S6.1 but for different years. 